\title{
Search for supersymmetry with R-parity violating LLE $\bar{E}$ couplings at $\sqrt{s}=183 \mathrm{GeV}$
}

DELPHI Collaboration

\begin{abstract}
Searches for pair production of supersymmetric particles in $\mathrm{e}^{+} \mathrm{e}^{-}$collisions at centre-of-mass energy of $183 \mathrm{GeV}$ have been performed on DELPHI data under the assumption that $R$-parity is not conserved. Only one $R$-parity violating coupling of $\lambda$ type, which couples the sleptons to the leptons ( $L L \bar{E}$ term), is considered to be dominant at a time. Since in models with $R$-parity violation any supersymmetric particle can be the lightest one, searches for charginos, neutralinos, sleptons and squarks have been performed both for direct $R$-parity violating decays and for indirect cascade decays. Morever, it is assumed that the strength of the $R$-parity violating couplings is such that the lifetimes can be neglected. The present study of supersymmetric particle pair production is used to exclude domains of the Minimal Supersymmetric Standard Model parameter space previously explored under the assumption of $R$-parity conservation.
\end{abstract}




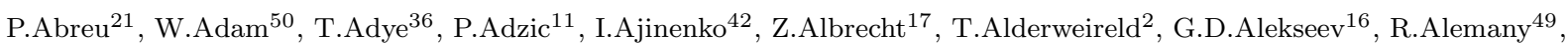
T.Allmendinger ${ }^{17}$, P.P.Allport ${ }^{22}$, S.Almehed ${ }^{24}$, U.Amaldi ${ }^{9}$, N.Amapane ${ }^{45}$, S.Amato ${ }^{47}$, E.G.Anassontzis ${ }^{3}$, P.Andersson ${ }^{44}$, A.Andreazza ${ }^{9}$, S.Andringa ${ }^{21}$, P.Antilogus ${ }^{25}$, W-D.Apel ${ }^{17}$, Y.Arnoud ${ }^{9}$, B.Åsman ${ }^{44}$, J-E.Augustin ${ }^{25}$, A.Augustinus ${ }^{9}$, P.Baillon $^{9}$, P.Bambade ${ }^{19}$, F.Barao ${ }^{21}$, G.Barbiellini ${ }^{46}$, R.Barbier ${ }^{25}$, D.Y.Bardin ${ }^{16}$, G.Barker ${ }^{17}, \quad$ A.Baroncelli ${ }^{38}$, M.Battaglia ${ }^{15}$, M.Baubillier ${ }^{23}$, K-H.Becks ${ }^{52}$, M.Begalli ${ }^{6}$, A.Behrmann ${ }^{52}$, P.Beilliere ${ }^{8}$, Yu.Belokopytov ${ }^{9,53}$, N.C.Benekos ${ }^{31}$, A.C.Benvenuti ${ }^{5}$, C.Berat ${ }^{14}$, M.Berggren ${ }^{25}$, D.Bertini ${ }^{25}$, D.Bertrand ${ }^{2}$, M.Besancon ${ }^{39}$, M.Bigi ${ }^{45}$, M.S.Bilenky ${ }^{16}$, M-A.Bizouard ${ }^{19}$, D.Bloch ${ }^{10}, \quad$ H.M.Blom ${ }^{30}, \quad$ M.Bonesini ${ }^{27}, \quad$ W.Bonivento ${ }^{27}, \quad$ M.Boonekamp ${ }^{39}, \quad$ P.S.L.Booth ${ }^{22}$, A.W.Borgland ${ }^{4}$, G.Borisov ${ }^{19}$, C.Bosio ${ }^{41}$, O.Botner ${ }^{48}$, E.Boudinov ${ }^{30}$, B.Bouquet ${ }^{19}$, C.Bourdarios ${ }^{19}$, T.J.V.Bowcock ${ }^{22}$, I.Boyko $^{16}$, I.Bozovic ${ }^{11}$, M.Bozzo ${ }^{13}$, P.Branchini ${ }^{38}$, T.Brenke ${ }^{52}$, R.A.Brenner ${ }^{48}$, P.Bruckman ${ }^{18}$, J-M.Brunet ${ }^{8}$, L.Bugge ${ }^{32}$, T.Buran $^{32}$, T.Burgsmueller ${ }^{52}$, B.Buschbeck ${ }^{50}$, P.Buschmann ${ }^{52}$, S.Cabrera ${ }^{49}$, M.Caccia ${ }^{27}$, M.Calvi ${ }^{27}$, T.Camporesi ${ }^{9}$, V.Canale ${ }^{37}$, F.Carena ${ }^{9}$, L.Carroll ${ }^{22}$, C.Caso ${ }^{13}$, M.V.Castillo Gimenez ${ }^{49}$, A.Cattai ${ }^{9}$, F.R.Cavallo ${ }^{5}$, V.Chabaud ${ }^{9}$, Ph.Charpentier $^{9}$, L.Chaussard ${ }^{25}$, P.Checchia ${ }^{35}$, G.A.Chelkov ${ }^{16}, \quad$ R.Chierici ${ }^{45}$, P.Chliapnikov ${ }^{42}, \quad$ P.Chochula ${ }^{7}$, V.Chorowicz ${ }^{25}$, J.Chudoba ${ }^{29}$, K.Cieslik ${ }^{18}$, P.Collins ${ }^{9}$, R.Contri ${ }^{13}$, E.Cortina ${ }^{49}$, G.Cosme ${ }^{19}$, F.Cossutti ${ }^{9}$, J-H.Cowell ${ }^{22}$, H.B.Crawley ${ }^{1}$, D.Crennell ${ }^{36}$, S.Crepe ${ }^{14}$, G.Crosetti ${ }^{13}$, J.Cuevas Maestro ${ }^{33}$, S.Czellar ${ }^{15}$, M.Davenport ${ }^{9}$, W.Da Silva ${ }^{23}$, A.Deghorain ${ }^{2}$, G.Della Ricca ${ }^{46}$, P.Delpierre ${ }^{26}$, N.Demaria ${ }^{9}$, A.De Angelis ${ }^{9}$, W.De Boer ${ }^{17}$, C.De Clercq ${ }^{2}$, B.De Lotto ${ }^{46}$, A.De Min ${ }^{35}$, L.De Paula ${ }^{47}$, H.Dijkstra ${ }^{9}$, L.Di Ciaccio ${ }^{37,9}$, J.Dolbeau ${ }^{8}$, K.Doroba ${ }^{51}$, M.Dracos ${ }^{10}$, J.Drees ${ }^{52}$, M.Dris ${ }^{31}$, A.Duperrin ${ }^{25}$, J-D.Durand ${ }^{9}$, G.Eigen ${ }^{4}$, T.Ekelof ${ }^{48}$, G.Ekspong ${ }^{44}$, M.Ellert ${ }^{48}$, M.Elsing ${ }^{9}$, J-P.Engel ${ }^{10}$, B.Erzen ${ }^{43}$, M.Espirito Santo ${ }^{21}$, E.Falk ${ }^{24}$, G.Fanourakis ${ }^{11}$, D.Fassouliotis ${ }^{11}$, J.Fayot ${ }^{23}$, M.Feindt ${ }^{17}$, P.Ferrari ${ }^{27}$, A.Ferrer ${ }^{49}$, E.Ferrer-Ribas ${ }^{19}$, F.Ferro ${ }^{13}$, S.Fichet ${ }^{23}$, A.Firestone ${ }^{1}$, U.Flagmeyer ${ }^{52}$, H.Foeth ${ }^{9}$, E.Fokitis ${ }^{31}$, F.Fontanelli ${ }^{13}$, B.Franek ${ }^{36}$, A.G.Frodesen ${ }^{4}$, R.Fruhwirth ${ }^{50}$, F.Fulda-Quenzer ${ }^{19}$, J.Fuster ${ }^{49}$, A.Galloni ${ }^{22}$, D.Gamba ${ }^{45}$, S.Gamblin ${ }^{19}$, M.Gandelman ${ }^{47}$, C.Garcia $^{49}$, C.Gaspar ${ }^{9}, \quad$ M.Gaspar ${ }^{47}, \quad$ U.Gasparini $^{35}, \quad$ Ph.Gavillet $^{9}, \quad$ E.N.Gazis ${ }^{31}, \quad$ D.Gele ${ }^{10}, \quad$ L.Gerdyukov $^{42}$, N.Ghodbane ${ }^{25}$, I.Gil ${ }^{49}$, F.Glege ${ }^{52}$, R.Gokieli ${ }^{9,51}$, B.Golob ${ }^{43}$, G.Gomez-Ceballos ${ }^{40}$, P.Goncalves ${ }^{21}$, I.Gonzalez Caballero ${ }^{40}$, G.Gopal $^{36}$, L.Gorn ${ }^{1,54}$, M.Gorski ${ }^{51}$, Yu.Gouz ${ }^{42}$, V.Gracco ${ }^{13}$, J.Grahl ${ }^{1}$, E.Graziani ${ }^{38}$, C.Green ${ }^{22}$, H-J.Grimm ${ }^{17}$, P.Gris $^{39}$, G.Grosdidier ${ }^{19}$, K.Grzelak ${ }^{51}$, M.Gunther ${ }^{48}$, J.Guy ${ }^{36}$, F.Hahn ${ }^{9}$, S.Hahn ${ }^{52}$, S.Haider ${ }^{9}$, A.Hallgren ${ }^{48}$, K.Hamacher ${ }^{52}$, J.Hansen $^{32}$, F.J.Harris ${ }^{34}$, V.Hedberg ${ }^{24}$, S.Heising ${ }^{17}$, J.J.Hernandez ${ }^{49}, \quad$ P.Herquet $^{2}$, H.Herr ${ }^{9}, \quad$ T.L.Hessing ${ }^{34}$, J.-M.Heuser ${ }^{52}$, E.Higon ${ }^{49}$, S-O.Holmgren ${ }^{44}$, P.J.Holt ${ }^{34}, \quad$ S.Hoorelbeke ${ }^{2}, \quad$ M.Houlden ${ }^{22}$, J.Hrubec ${ }^{50}, \quad$ K.Huet ${ }^{2}$, G.J.Hughes $^{22}$, K.Hultqvist ${ }^{44}$, J.N.Jackson ${ }^{22}$, R.Jacobsson ${ }^{9}$, P.Jalocha ${ }^{9}$, R.Janik ${ }^{7}$, Ch.Jarlskog ${ }^{24}$, G.Jarlskog ${ }^{24}$, P.Jarry ${ }^{39}$, B.Jean-Marie ${ }^{19}$, E.K.Johansson ${ }^{44}$, P.Jonsson ${ }^{25}$, C.Joram ${ }^{9}$, P.Juillot ${ }^{10}$, F.Kapusta ${ }^{23}$, K.Karafasoulis ${ }^{11}$, S.Katsanevas ${ }^{25}$, E.C.Katsoufis ${ }^{31}$, R.Keranen ${ }^{17}$, B.P.Kersevan ${ }^{43}$, B.A.Khomenko ${ }^{16}$, N.N.Khovanski ${ }^{16}$, A.Kiiskinen ${ }^{15}, \quad$ B.King ${ }^{22}$, A.Kinvig ${ }^{22}$, N.J.Kjaer ${ }^{30}$, O.Klapp ${ }^{52}$, H.Klein ${ }^{9}, \quad$ P.Kluit ${ }^{30}, \quad$ P.Kokkinias ${ }^{11}, \quad$ M.Koratzinos ${ }^{9}, \quad$ V.Kostioukhine $^{42}$, C.Kourkoumelis ${ }^{3}$, O.Kouznetsov ${ }^{39}$, M.Krammer ${ }^{50}$, E.Kriznic ${ }^{43}$, P.Krstic ${ }^{11}$, Z.Krumstein ${ }^{16}$, P.Kubinec ${ }^{7}$, J.Kurowska ${ }^{51}$, K.Kurvinen $^{15}$, J.W.Lamsa ${ }^{1}$, D.W.Lane ${ }^{1}$, P.Langefeld ${ }^{52}$, V.Lapin ${ }^{42}$, J-P.Laugier ${ }^{39}$, R.Lauhakangas ${ }^{15}$, G.Leder ${ }^{50}$, F.Ledroit ${ }^{14}$, V.Lefebure ${ }^{2}$, L.Leinonen ${ }^{44}$, A.Leisos ${ }^{11}$, R.Leitner ${ }^{29}$, G.Lenzen ${ }^{52}$, V.Lepeltier ${ }^{19}$, T.Lesiak ${ }^{18}$, M.Lethuillier ${ }^{39}$, J.Libby $^{34}$, D.Liko ${ }^{9}, \quad$ A.Lipniacka ${ }^{44}$, I.Lippi ${ }^{35}$, B.Loerstad ${ }^{24}$, J.G.Loken ${ }^{34}$, J.H.Lopes ${ }^{47}$, J.M.Lopez ${ }^{40}$, R.Lopez-Fernandez ${ }^{14}$, D.Loukas ${ }^{11}$, P.Lutz ${ }^{39}$, L.Lyons ${ }^{34}$, J.R.Mahon ${ }^{6}$, A.Maio ${ }^{21}$, A.Malek ${ }^{52}$, T.G.M.Malmgren ${ }^{44}$, S.Maltezos $^{31}$, V.Malychev ${ }^{16}$, F.Mandl ${ }^{50}$, J.Marco ${ }^{40}$, R.Marco ${ }^{40}$, B.Marechal ${ }^{47}$, M.Margoni ${ }^{35}$, J-C.Marin ${ }^{9}$, C.Mariotti ${ }^{9}$, A.Markou $^{11}$, C.Martinez-Rivero ${ }^{19}, \quad$ F.Martinez-Vidal ${ }^{49}, \quad$ S.Marti i Garcia ${ }^{9}, \quad$ J.Masik $^{12}, \quad$ N.Mastroyiannopoulos ${ }^{11}$, F.Matorras ${ }^{40}$, C.Matteuzzi ${ }^{27}$, G.Matthiae ${ }^{37}, \quad$ F.Mazzucato ${ }^{35}, \quad$ M.Mazzucato $^{35}$, M.Mc Cubbin ${ }^{22}, \quad$ R.Mc Kay ${ }^{1}$, R.Mc Nulty ${ }^{22}$, G.Mc Pherson ${ }^{22}$, C.Meroni ${ }^{27}$, W.T.Meyer ${ }^{1}$, A.Miagkov ${ }^{42}$, E.Migliore ${ }^{45}$, L.Mirabito ${ }^{25}$, W.A.Mitaroff ${ }^{50}$, U.Mjoernmark $^{24}$, T.Moa ${ }^{44}$, M.Moch ${ }^{17}$, R.Moeller ${ }^{28}$, K.Moenig ${ }^{9}$, M.R.Monge ${ }^{13}$, X.Moreau ${ }^{23}$, P.Morettini ${ }^{13}$, G.Morton ${ }^{34}$, U.Mueller $^{52}$, K.Muenich ${ }^{52}$, M.Mulders ${ }^{30}$, C.Mulet-Marquis ${ }^{14}$, R.Muresan ${ }^{24}$, W.J.Murray ${ }^{36}$, B.Muryn ${ }^{14,18}$, G.Myatt ${ }^{34}$, T.Myklebust ${ }^{32}$, F.Naraghi ${ }^{14}$, M.Nassiakou ${ }^{11}$, F.L.Navarria ${ }^{5}$, S.Navas ${ }^{49}$, K.Nawrocki ${ }^{51}$, P.Negri ${ }^{27}$, S.Nemecek ${ }^{12}$, N.Neufeld ${ }^{9}$, N.Neumeister ${ }^{50}$, R.Nicolaidou ${ }^{39}$, B.S.Nielsen ${ }^{28}, \quad$ M.Nikolenko ${ }^{10,16}$, V.Nomokonov ${ }^{15}$, A.Normand $^{22}$,

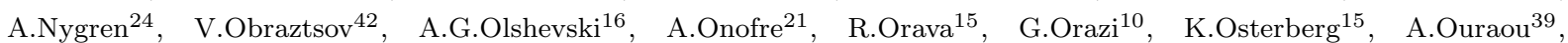
M.Paganoni ${ }^{27}$, S.Paiano ${ }^{5}$, R.Pain ${ }^{23}$, R.Paiva ${ }^{21}$, J.Palacios ${ }^{34}$, H.Palka ${ }^{18}$, Th.D.Papadopoulou ${ }^{31,9}$, K.Papageorgiou ${ }^{11}$, L.Pape $^{9}$, C.Parkes ${ }^{9}$, F.Parodi ${ }^{13}$, U.Parzefall ${ }^{22}$, A.Passeri ${ }^{38}$, O.Passon ${ }^{52}$, M.Pegoraro ${ }^{35}$, L.Peralta ${ }^{21}$, M.Pernicka ${ }^{50}$, A.Perrotta $^{5}$, C.Petridou ${ }^{46}$, A.Petrolini ${ }^{13}$, H.T.Phillips ${ }^{36}$, F.Pierre ${ }^{39}$, M.Pimenta ${ }^{21}$, E.Piotto ${ }^{27}$, T.Podobnik ${ }^{43}$, M.E.Pol ${ }^{6}$, G.Polok $^{18}$, P.Poropat ${ }^{46}$, V.Pozdniakov ${ }^{16}$, P.Privitera ${ }^{37}$, N.Pukhaeva ${ }^{16}$, A.Pullia ${ }^{27}$, D.Radojicic ${ }^{34}$, S.Ragazzi $^{27}$, H.Rahmani ${ }^{31}$, P.N.Ratoff ${ }^{20}$, A.L.Read ${ }^{32}, \quad$ P.Rebecchi ${ }^{9}, \quad$ N.G.Redaelli ${ }^{27}, \quad$ M.Regler ${ }^{50}, \quad$ D.Reid ${ }^{30}, \quad$ R.Reinhardt ${ }^{52}$, P.B.Renton ${ }^{34}$, L.K.Resvanis ${ }^{3}$, F.Richard ${ }^{19}, \quad$ J.Ridky $^{12}$, G.Rinaudo ${ }^{45}, \quad$ O.Rohne $^{32}$, A.Romero ${ }^{45}, \quad$ P.Ronchese ${ }^{35}$, E.I.Rosenberg ${ }^{1}$, P.Rosinsky ${ }^{7}$, P.Roudeau ${ }^{19}$, T.Rovelli ${ }^{5}$, Ch.Royon ${ }^{39}$, V.Ruhlmann-Kleider ${ }^{39}$, A.Ruiz ${ }^{40}$, H.Saarikko ${ }^{15}$, Y.Sacquin ${ }^{39}$, A.Sadovsky ${ }^{16}$, G.Sajot ${ }^{14}$, J.Salt ${ }^{49}$, D.Sampsonidis ${ }^{11}$, M.Sannino ${ }^{13}$, H.Schneider ${ }^{17}$, Ph.Schwemling ${ }^{23}$, B.Schwering ${ }^{52}$, U.Schwickerath ${ }^{17}$, M.A.E.Schyns ${ }^{52}$, F.Scuri ${ }^{46}$, P.Seager ${ }^{20}$, Y.Sedykh ${ }^{16}$, A.M.Segar ${ }^{34}$, R.Sekulin ${ }^{36}$, R.C.Shellard ${ }^{6}$, A.Sheridan ${ }^{22}$, M.Siebel ${ }^{52}$, L.Simard ${ }^{39}$, F.Simonetto ${ }^{35}$, A.N.Sisakian ${ }^{16}$, G.Smadja ${ }^{25}$, N.Smirnov ${ }^{42}$, O.Smirnova ${ }^{24}$, G.R.Smith ${ }^{36}$, O.Solovianov ${ }^{42}$, A.Sopczak ${ }^{17}$, R.Sosnowski ${ }^{51}$, T.Spassov ${ }^{21}$, E.Spiriti ${ }^{38}$, P.Sponholz ${ }^{52}$, S.Squarcia ${ }^{13}$, C.Stanescu ${ }^{38}$, S.Stanic ${ }^{43}$, K.Stevenson ${ }^{34}$, A.Stocchi ${ }^{19}$, J.Strauss ${ }^{50}$, R.Strub ${ }^{10}$, B.Stugu ${ }^{4}$, M.Szczekowski ${ }^{51}$, M.Szeptycka ${ }^{51}$, T.Tabarelli ${ }^{27}$, F.Tegenfeldt ${ }^{48}$, F.Terranova ${ }^{27}$, J.Thomas ${ }^{34}$, J.Timmermans ${ }^{30}$, N.Tinti ${ }^{5}$, L.G.Tkatchev ${ }^{16}$, S.Todorova ${ }^{10}$, A.Tomaradze ${ }^{2}$, B.Tome ${ }^{21}$, A.Tonazzo ${ }^{9}$, L.Tortora ${ }^{38}$, G.Transtromer ${ }^{24}$, D.Treille ${ }^{9}$, G.Tristram ${ }^{8}$, 
M.Trochimczuk ${ }^{51}$, C.Troncon ${ }^{27}$, A.Tsirou ${ }^{9}$, M-L.Turluer ${ }^{39}$, I.A.Tyapkin ${ }^{16}$, S.Tzamarias ${ }^{11}$, O.Ullaland ${ }^{9}$, V.Uvarov ${ }^{42}$, G.Valenti ${ }^{5}$, E.Vallazza ${ }^{46}$, G.W.Van Apeldoorn ${ }^{30}$, P.Van Dam ${ }^{30}$, J.Van Eldik ${ }^{30}$, A.Van Lysebetten ${ }^{2}$, N.Van Remortel ${ }^{2}$, I.Van Vulpen ${ }^{30}$, N.Vassilopoulos ${ }^{34}$, G.Vegni ${ }^{27}$, L.Ventura ${ }^{35}$, W.Venus ${ }^{36,9}$, F.Verbeure ${ }^{2}$, M.Verlato ${ }^{35}$, L.S.Vertogradov ${ }^{16}$, V.Verzi $^{37}$, D.Vilanova ${ }^{39}$, L.Vitale ${ }^{46}$, E.Vlasov ${ }^{42}$, A.S.Vodopyanov ${ }^{16}$, C.Vollmer ${ }^{17}$, G.Voulgaris ${ }^{3}$, V.Vrba ${ }^{12}$, H.Wahlen ${ }^{52}$, C.Walck ${ }^{44}$, C.Weiser ${ }^{17}$, D.Wicke ${ }^{52}$, J.H.Wickens ${ }^{2}$, G.R.Wilkinson ${ }^{9}, \quad$ M.Winter ${ }^{10}, \quad$ M.Witek ${ }^{18}, \quad$ G.Wolf ${ }^{9}, \quad$ J.Yi $^{1}$, O.Yushchenko $^{42}$, A.Zalewska ${ }^{18}$, P.Zalewski ${ }^{51}$, D.Zavrtanik ${ }^{43}$, E.Zevgolatakos ${ }^{11}$, N.I.Zimin ${ }^{16,24}$, G.C.Zucchelli ${ }^{44}$, G.Zumerle ${ }^{35}$

${ }^{1}$ Department of Physics and Astronomy, Iowa State University, Ames IA 50011-3160, USA

${ }^{2}$ Physics Department, Univ. Instelling Antwerpen, Universiteitsplein 1, BE-2610 Wilrijk, Belgium and IIHE, ULB-VUB, Pleinlaan 2, BE-1050 Brussels, Belgium

and Faculté des Sciences, Univ. de l'Etat Mons, Av. Maistriau 19, BE-7000 Mons, Belgium

${ }^{3}$ Physics Laboratory, University of Athens, Solonos Str. 104, GR-10680 Athens, Greece

${ }^{4}$ Department of Physics, University of Bergen, Allégaten 55, NO-5007 Bergen, Norway

${ }^{5}$ Dipartimento di Fisica, Università di Bologna and INFN, Via Irnerio 46, IT-40126 Bologna, Italy

${ }^{6}$ Centro Brasileiro de Pesquisas Físicas, rua Xavier Sigaud 150, BR-22290 Rio de Janeiro, Brazil and Depto. de Física, Pont. Univ. Católica, C.P. 38071 BR-22453 Rio de Janeiro, Brazil

and Inst. de Física, Univ. Estadual do Rio de Janeiro, rua São Francisco Xavier 524, Rio de Janeiro, Brazil

${ }^{7}$ Comenius University, Faculty of Mathematics and Physics, Mlynska Dolina, SK-84215 Bratislava, Slovakia

${ }^{8}$ Collège de France, Lab. de Physique Corpusculaire, IN2P3-CNRS, FR-75231 Paris Cedex 05, France

${ }^{9} \mathrm{CERN}, \mathrm{CH}-1211$ Geneva 23, Switzerland

${ }^{10}$ Institut de Recherches Subatomiques, IN2P3 - CNRS/ULP - BP20, FR-67037 Strasbourg Cedex, France

${ }^{11}$ Institute of Nuclear Physics, N.C.S.R. Demokritos, P.O. Box 60228, GR-15310 Athens, Greece

${ }^{12}$ FZU, Inst. of Phys. of the C.A.S. High Energy Physics Division, Na Slovance 2, CZ-180 40, Praha 8, Czech Republic

${ }^{13}$ Dipartimento di Fisica, Università di Genova and INFN, Via Dodecaneso 33, IT-16146 Genova, Italy

${ }^{14}$ Institut des Sciences Nucléaires, IN2P3-CNRS, Université de Grenoble 1, FR-38026 Grenoble Cedex, France

${ }^{15}$ Helsinki Institute of Physics, HIP, P.O. Box 9, FI-00014 Helsinki, Finland

${ }^{16}$ Joint Institute for Nuclear Research, Dubna, Head Post Office, P.O. Box 79, RU-101 000 Moscow, Russian Federation

${ }^{17}$ Institut für Experimentelle Kernphysik, Universität Karlsruhe, Postfach 6980, DE-76128 Karlsruhe, Germany

${ }^{18}$ Institute of Nuclear Physics and University of Mining and Metalurgy, Ul. Kawiory 26a, PL-30055 Krakow, Poland

${ }^{19}$ Université de Paris-Sud, Lab. de l'Accélérateur Linéaire, IN2P3-CNRS, Bât. 200, FR-91405 Orsay Cedex, France

${ }^{20}$ School of Physics and Chemistry, University of Lancaster, Lancaster LA1 4YB, UK

${ }^{21}$ LIP, IST, FCUL - Av. Elias Garcia, 14-1 ${ }^{\circ}$, PT-1000 Lisboa Codex, Portugal

${ }^{22}$ Department of Physics, University of Liverpool, P.O. Box 147, Liverpool L69 3BX, UK

${ }^{23}$ LPNHE, IN2P3-CNRS, Univ. Paris VI et VII, Tour 33 (RdC), 4 place Jussieu, FR-75252 Paris Cedex 05, France

${ }^{24}$ Department of Physics, University of Lund, Sölvegatan 14, SE-223 63 Lund, Sweden

${ }^{25}$ Université Claude Bernard de Lyon, IPNL, IN2P3-CNRS, FR-69622 Villeurbanne Cedex, France

${ }^{26}$ Univ. d'Aix - Marseille II - CPP, IN2P3-CNRS, FR-13288 Marseille Cedex 09, France

${ }^{27}$ Dipartimento di Fisica, Università di Milano and INFN, Via Celoria 16, IT-20133 Milan, Italy

${ }^{28}$ Niels Bohr Institute, Blegdamsvej 17, DK-2100 Copenhagen $\varnothing$, Denmark

${ }^{29}$ NC, Nuclear Centre of MFF, Charles University, Areal MFF, V Holesovickach 2, CZ-180 00, Praha 8, Czech Republic

${ }^{30}$ NIKHEF, Postbus 41882, NL-1009 DB Amsterdam, The Netherlands

${ }^{31}$ National Technical University, Physics Department, Zografou Campus, GR-15773 Athens, Greece

${ }^{32}$ Physics Department, University of Oslo, Blindern, NO-1000 Oslo 3, Norway

${ }^{33}$ Dpto. Fisica, Univ. Oviedo, Avda. Calvo Sotelo s/n, ES-33007 Oviedo, Spain

${ }^{34}$ Department of Physics, University of Oxford, Keble Road, Oxford OX1 3RH, UK

${ }^{35}$ Dipartimento di Fisica, Università di Padova and INFN, Via Marzolo 8, IT-35131 Padua, Italy

${ }^{36}$ Rutherford Appleton Laboratory, Chilton, Didcot OX11 OQX, UK

${ }^{37}$ Dipartimento di Fisica, Università di Roma II and INFN, Tor Vergata, IT-00173 Rome, Italy

${ }^{38}$ Dipartimento di Fisica, Università di Roma III and INFN, Via della Vasca Navale 84, IT-00146 Rome, Italy

${ }^{39}$ DAPNIA/Service de Physique des Particules, CEA-Saclay, FR-91191 Gif-sur-Yvette Cedex, France

${ }^{40}$ Instituto de Fisica de Cantabria (CSIC-UC), Avda. los Castros s/n, ES-39006 Santander, Spain

${ }^{41}$ Dipartimento di Fisica, Università degli Studi di Roma La Sapienza, Piazzale Aldo Moro 2, IT-00185 Rome, Italy

${ }^{42}$ Inst. for High Energy Physics, Serpukov P.O. Box 35, Protvino, (Moscow Region), Russian Federation

${ }^{43}$ J. Stefan Institute, Jamova 39, SI-1000 Ljubljana, Slovenia and Laboratory for Astroparticle Physics,

Nova Gorica Polytechnic, Kostanjeviska 16a, SI-5000 Nova Gorica, Slovenia,

and Department of Physics, University of Ljubljana, SI-1000 Ljubljana, Slovenia

${ }^{44}$ Fysikum, Stockholm University, Box 6730, SE-113 85 Stockholm, Sweden

${ }^{45}$ Dipartimento di Fisica Sperimentale, Università di Torino and INFN, Via P. Giuria 1, IT-10125 Turin, Italy

${ }^{46}$ Dipartimento di Fisica, Università di Trieste and INFN, Via A. Valerio 2, IT-34127 Trieste, Italy

and Istituto di Fisica, Università di Udine, IT-33100 Udine, Italy

${ }^{47}$ Univ. Federal do Rio de Janeiro, C.P. 68528 Cidade Univ., Ilha do Fundão BR-21945-970 Rio de Janeiro, Brazil

${ }^{48}$ Department of Radiation Sciences, University of Uppsala, P.O. Box 535, SE-751 21 Uppsala, Sweden

${ }^{49}$ IFIC, Valencia-CSIC, and D.F.A.M.N., U. de Valencia, Avda. Dr. Moliner 50, ES-46100 Burjassot (Valencia), Spain

${ }^{50}$ Institut für Hochenergiephysik, Österr. Akad. d. Wissensch., Nikolsdorfergasse 18, AT-1050 Vienna, Austria

${ }^{51}$ Inst. Nuclear Studies and University of Warsaw, Ul. Hoza 69, PL-00681 Warsaw, Poland

${ }^{52}$ Fachbereich Physik, University of Wuppertal, Postfach 100 127, DE-42097 Wuppertal, Germany

${ }^{53} \mathrm{On}$ leave of absence from IHEP Serpukhov

${ }^{54}$ Now at University of Florida 


\section{Introduction}

This paper presents the searches for pair produced neutralinos, charginos and sfermions performed in the data sample collected by the DELPHI detector at centre-of-mass energy of $183 \mathrm{GeV}$, in the hypothesis of $R$-parity violation with only one dominant $\lambda_{i j k}$ coupling, which couples the sleptons to the leptons.

\subsection{The $R$-parity violating lagrangian}

The construction of a supersymmetric extension of the Standard Model leads to a lagrangian containing terms allowed by the $S U(3) \times S U(2) \times U(1)$ gauge symmetry which violate either baryon number $(B)$ or lepton number $(L)$ [1]. If present, these terms would induce a proton decay rate larger by many orders of magnitude than the experimental limits. In the Minimal Supersymmetric extension of the Standard Model (MSSM) $[2,3]$, one adds a new discrete symmetry which eliminates the possibility to have such $B$ and $L$ violating terms in the superpotential. This new symmetry, called " $R$-parity", can be translated in a multiplicatively conserved quantum number $R_{p}$ defined as $R_{p}=(-1)^{3 B+L+2 S}$ for a particle with spin $S$ [4]. Standard particles have even $R$-parity, and the corresponding superpartners have odd $R$-parity. The MSSM is designed to conserve $R$-parity: it is phenomenologically justified by proton decay constraints, and by the hope that a neutral Lightest Supersymmetric Particle (LSP) will provide a good dark matter candidate. However, from a theoretical point of view, $R$-parity conservation is not needed in supersymmetric extensions of the Standard Model.

Retaining the same minimal particle content as in the MSSM, a more general superpotential containing the three following terms [5]:

$$
\lambda_{i j k} L_{i} L_{j} \bar{E}_{k}+\lambda_{i j k}^{\prime} L_{i} Q_{j} \bar{D}_{k}+\lambda_{i j k}^{\prime \prime} \bar{U}_{i} \bar{D}_{j} \bar{D}_{k}
$$

is obtained by removing its invariance with respect to the $R$-parity symmetry. In the above formula, $i, j$ and $k$ are the generation indices, $L(Q)$ denote the lepton (quark) doublet superfields, $\bar{E}(\bar{U}, \bar{D})$ denote the lepton (up and down quark) singlet superfields, $\lambda_{i j k}, \lambda_{i j k}^{\prime}$ and $\lambda_{i j k}^{\prime \prime}$ are Yukawa couplings. The two first terms violate $L$ conservation, and the third one $B$ conservation. Since $\lambda_{i j k}=-\lambda_{j i k}, \lambda_{i j k}^{\prime \prime}=-\lambda_{i k j}^{\prime \prime}$, there are $9 \lambda_{i j k}, 27 \lambda_{i j k}^{\prime}$ and $9 \lambda_{i k j}^{\prime \prime}$ leading to 45 additional couplings. Nevertheless, as already mentioned above, all $R$-parity violating $\left(R_{p}\right)$ terms cannot be simultaneously present without contradicting present experimental limit on proton lifetime $[6,7]$.

One major phenomenological consequence of the $R$-parity violation is that the LSP is allowed to decay to standard fermions. This fact modifies the signatures of supersymmetric particle production compared to those expected in the case of $R$-parity conservation. Moreover, single sparticle production is possible [8], under more restrictive conditions than pair production, in particular on the $R$-parity violating coupling strength involved not only in the sparticle decay but also in the resonant cross-section.

\subsection{Pair production of supersymmetric particles}

In the MSSM, the masses and mixing angles of the neutralinos and the charginos are determined by the values of the four parameters $M_{1}, M_{2}$, the $\mathrm{U}(1)$ and $\mathrm{SU}(2)$ gaugino mass parameters at the electroweak scale, $\mu$, the mixing mass term of the Higgs doublets at the electroweak scale and $\tan \beta$, the ratio of the vacuum expectation values of the two Higgs doublets. The assumption that the gaugino masses are unified at the GUT scale implies $M_{1}=\frac{5}{3} \tan ^{2} \theta_{W} M_{2} \simeq \frac{1}{2} M_{2}$ at the electroweak scale. 
The neutralinos and the charginos are produced by pairs in the $s$-channel via $\gamma$ or Z exchange, or via $t$-channel exchange of a selectron (sneutrino) for the neutralinos (charginos) (Fig. 1). The $t$-channel exchange is suppressed when the slepton masses are high enough. It is also assumed that $m_{0}$, the scalar common mass at the GUT scale, determines the slepton masses. When the selectron mass is sufficiently small (less than $100 \mathrm{GeV} / c^{2}$ ), the neutralino production can be enhanced, because of the $t$-channel ẽ exchange contribution. On the contrary, if the $\widetilde{\nu}_{\mathrm{e}}$ mass is in the same range, the chargino cross-section can decrease due to destructive interference between the $s$ - and $t$-channel amplitudes. If the dominant component of neutralinos and charginos is the higgsino $\left(|\mu| \ll M_{2}\right)$, the production cross-sections are large and insensitive to slepton masses. The appropriate MSSM parameters to consider in the general scan are then $M_{2}, \mu, \tan \beta$ and $m_{0}$. Depending on the values of the different parameters, the cross-sections at $\sqrt{s}=183 \mathrm{GeV}$ vary typically from 0.1 to $10 \mathrm{pb}$.

The sfermion mass-eigenstates, $\tilde{\mathrm{f}}_{1}$ and $\tilde{\mathrm{f}}_{2}$ (f: $\mathrm{q}$ or $l, \tilde{\mathrm{f}}_{1}$ lighter than $\tilde{\mathrm{f}}_{2}$ ), are obtained from the two supersymmetric scalar partners $\tilde{\mathrm{f}}_{L}$ and $\tilde{\mathrm{f}}_{R}$ of the corresponding left and right-handed fermion $[9,10]$ :

$$
\begin{aligned}
& \tilde{\mathrm{f}}_{1}=\tilde{\mathrm{f}}_{L} \cos \phi_{\tilde{\mathrm{f}}}+\tilde{\mathrm{f}}_{R} \sin \phi_{\tilde{\mathrm{f}}} \\
& \tilde{\mathrm{f}}_{2}=-\tilde{\mathrm{f}}_{L} \sin \phi_{\tilde{\mathrm{f}}}+\tilde{\mathrm{f}}_{R} \cos \phi_{\tilde{\mathrm{f}}}
\end{aligned}
$$

where $\phi_{\tilde{\mathrm{f}}}$ is the mixing angle with $0 \leq \phi_{\tilde{\mathrm{f}}} \leq \pi$. According to the equations which give the sfermion masses (see for example in [3]), the left-handed sfermions are likely to be heavier than their right-handed counterparts. The $\tilde{\mathrm{f}}_{L}-\tilde{\mathrm{f}}_{R}$ mixing is related to the off-diagonal terms of the scalar squared-mass matrix. It is proportional to the fermion mass, and is small compared to the diagonal terms, with the possible exception of the third family sfermion [11]. The lighter stop $\tilde{t}_{1}$ is then probably the lightest squark. This is due not only to the mixing effect but also to the effect of the large top Yukawa coupling; both tend to decrease the mass of $\tilde{t}_{1}[12]$. The lightest charged slepton is probably the $\tilde{\tau}_{1}$. For small values of $\tan \beta, \tilde{\tau}_{1}$ is predominantly a $\tilde{\tau}_{R}$, and it is not so much lighter than $\tilde{e}_{R}$ and $\tilde{\mu}_{R}$.

Sfermions can be produced via $s$-channel $\mathrm{Z}$ or $\gamma$ exchange (the latter only in the case of charged sfermions) as shown in Fig. 1; the production cross-section depends on the sfermion mass. The $\widetilde{\nu}_{\mathrm{e}}(\tilde{\mathrm{e}})$ can also be produced via the exchange of a chargino (neutralino) in the $t$-channel, and then the cross-section depends also on the $\widetilde{\chi}^{ \pm}\left(\widetilde{\chi}^{0}\right)$ mass and field composition and thereby on the four MSSM parameters mentioned above. In the case of the third generation, the dependence of the sfermion-sfermion- $Z$ coupling on the mixing angle $\phi_{\tilde{\mathrm{f}}}$ has direct consequences on the cross-section. In particular, the $Z \tilde{t}_{1} \tilde{t}_{1}$ coupling vanishes for the mixing angle $\phi_{\tilde{\mathrm{t}}}=0.98 \mathrm{rad}[13]$.

\subsection{Decays of supersymmetric particles}

\section{- Direct decay}

In a direct decay the sparticle decays, either directly or via a sparticle virtual exchange, to standard particles through an $R_{p}$ vertex. A direct decay is the only possibility when the sparticle is the LSP. If for example the $\tilde{\nu}$ is the LSP, it can decay directly into a pair of charged leptons through the $\lambda_{i j k} R_{p}$ operators. If on the other hand the lightest neutralino $\tilde{\chi}_{1}^{0}$ is the LSP, then it can decay into a lepton and a virtual slepton with the subsequent decay of the virtual slepton to leptons via the $R$-parity violating $\lambda$ couplings (see Fig. 2-a,b). 
- Indirect decay

In an indirect decay the sparticle first decays through an $R_{p}$-conserving vertex to a standard particle and an on-shell sparticle which then decays through an $R_{p}$ vertex. A typical example is the $R_{p}$ decay $\tilde{\chi}_{1}^{+} \rightarrow \tilde{\chi}_{1}^{0}+\mathrm{W}^{*+}$ and the subsequent decay of $\tilde{\chi}_{1}^{0}$ through the $R_{p}$ couplings (see Fig. $2-\mathrm{d}$ ). The indirect decay mode usually dominates when there is enough phase space available in the decay between "mother" and "daughter" sparticles. When the difference of masses between these two sparticles is larger than $5-10 \mathrm{GeV}$ the indirect mode tends to dominate. Regions of the parameter space where one has a "dynamic" suppression of the $R_{p}$ conserving modes also exist. In this case, even if the sparticle is not the LSP, it decays through an $R_{p}$ mode. For example, if the field component of the two lightest neutralinos is mainly the photino, then the indirect decay $\widetilde{\chi}_{2}^{0} \rightarrow \widetilde{\chi}_{1}^{0} \mathrm{Z}^{*}$ is suppressed.

\section{- Neutralino and chargino decay}

In the case of a dominant $\lambda_{i j k}$ coupling, the sleptons couple to the leptons. The decay of the lightest neutralino leads to one neutrino and two charged leptons. The heavier neutralinos and the charginos, depending on their mass difference with $\widetilde{\chi}_{1}^{0}$, can either decay directly into three leptons, or decay to $\widetilde{\chi}_{1}^{0}$, via for example virtual $\mathrm{Z}$ or $\mathrm{W}$, as illustrated on Fig. 2 and in Table 1. Note that, even if the $\lambda$ couplings lead to purely leptonic decay modes of the lightest neutralino, the indirect decay of chargino or heavier neutralinos may contain hadrons in the final state depending on the decay modes of $\mathrm{W}^{*}$ and $\mathrm{Z}^{*}$. In order to cover both the direct and indirect decays of $\widetilde{\chi}_{i}^{0}$ and $\tilde{\chi}^{ \pm}$, the analysis has to be sensitive to the final states listed in Table 2 .

\begin{tabular}{|l|l|l|l|}
\hline Direct decay & $\widetilde{\chi}_{1}^{0} \rightarrow \nu l^{+} l^{-}$ & $\widetilde{\chi}_{2}^{0} \rightarrow \nu l^{+} l^{-}$ & $\widetilde{\chi}_{1}^{+} \rightarrow \nu \nu l^{+}, l^{+} l^{-} l^{+}$ \\
\hline Indirect decay & & $\widetilde{\chi}_{2}^{0} \rightarrow \mathrm{Z}^{*} \widetilde{\chi}_{1}^{0}, \mathrm{Z}^{*} \rightarrow f f$ & $\widetilde{\chi}_{1}^{+} \rightarrow W^{*+} \widetilde{\chi}_{1}^{0}, W^{*+} \rightarrow f f^{\prime}$ \\
& & $\widetilde{\chi}_{2}^{0} \rightarrow \widetilde{\chi}_{1}^{0} l^{+} l^{-}, \widetilde{\chi}_{1}^{0} \gamma$ & $\widetilde{\chi}_{1}^{+} \rightarrow \widetilde{\chi}_{1}^{0} \nu_{l} l^{+}$ \\
\hline
\end{tabular}

Table 1: Possible decays of neutralinos and charginos when a $\lambda$ coupling is dominant.

\begin{tabular}{|l|l|l|}
\hline Final states & \multicolumn{1}{|c|}{$\begin{array}{c}\text { Direct } \\
\text { decay of }\end{array}$} & $\begin{array}{c}\text { Indirect } \\
\text { decay of }\end{array}$ \\
\hline $2 l+E$ & $\widetilde{\chi}_{1}^{+} \widetilde{\chi}_{1}^{-}$ & \\
$4 l+E$ & $\widetilde{\chi}_{1}^{0} \widetilde{\chi}_{1}^{0}, \widetilde{\chi}_{1}^{+} \widetilde{\chi}_{1}^{-}$ & $\widetilde{\chi}_{2}^{0} \widetilde{\chi}_{1}^{0}$ \\
$6 l$ & $\widetilde{\chi}_{1}^{+} \widetilde{\chi}_{1}^{-}$ & \\
$6 l+E$ & & $\widetilde{\chi}_{1}^{+} \widetilde{\chi}_{1}^{-}, \widetilde{\chi}_{2}^{0} \widetilde{\chi}_{1}^{0}$ \\
$4 l+2$ jets $+E$ & & $\widetilde{\chi}_{2}^{0} \widetilde{\chi}_{1}^{0}$ \\
$4 l+4$ jets $+E$ & & $\widetilde{\chi}_{1}^{+} \widetilde{\chi}_{1}^{-}$ \\
$5 l+2$ jets $+E$ & & $\widetilde{\chi}_{1}^{+} \widetilde{\chi}_{1}^{-}$ \\
\hline
\end{tabular}

Table 2: Final states in $\tilde{\chi}_{i}^{0}, \tilde{\chi}^{ \pm}$pair production when a $\lambda$ coupling is dominant ( $E \equiv$ missing energy).

\section{- Sfermion decay}

A sneutrino with a mass lower than or very close to that of the $\widetilde{\chi}_{1}^{0}$ or $\tilde{\chi}^{ \pm}$mass, may decay directly to two charged leptons through an $\lambda_{i j k} L_{i} L_{j} \bar{E}_{k}$ operator (Fig. 3a). The possible decays are: $\tilde{\nu}_{i} \rightarrow l_{j}^{+} l_{k}^{-}$and $\tilde{\nu}_{j} \rightarrow l_{i}^{+} l_{k}^{-}$. If the sneutrino is not the LSP, the indirect decays $\tilde{\nu} \rightarrow \widetilde{\chi}_{1}^{0} \nu, \tilde{\nu} \rightarrow \widetilde{\chi}_{2}^{0} \nu, \tilde{\nu} \rightarrow \widetilde{\chi}^{ \pm} l^{\mp}$ are allowed (Fig. 3c), depending on the MSSM parameters; the $\tilde{\chi}_{2}^{0}$ and $\tilde{\chi}^{ \pm}$could also decay directly or indirectly, as previously 
explained. Furthermore the so-called "mixed decay" is possible when one $\tilde{\nu}$ in a pair decays directly and the other indirectly.

\begin{tabular}{|l|l|c|}
\hline \multicolumn{2}{|c|}{ Decay type } & Pair production signature \\
\hline Direct & $\widetilde{\nu} \rightarrow l^{+} l^{-}$ & $4 l$ \\
\hline Indirect & $\widetilde{\nu} \rightarrow \nu \widetilde{\chi}_{1}^{0}$ & $4 l+E$ \\
\cline { 2 - 4 } & $\widetilde{\nu} \rightarrow \nu \widetilde{\chi}_{2}^{0}$ & same as $\tilde{\chi}$ analyses \\
& $\widetilde{\nu} \rightarrow l^{ \pm} \widetilde{\chi}^{ \pm}$ & multilepton or lepton+jets \\
\hline Direct & $\bar{l}^{ \pm} \rightarrow l^{ \pm} \nu$ & $2 l($ acoplanar $)+\not E$ \\
\hline Indirect & $\widetilde{l}^{ \pm} \rightarrow l^{ \pm} \widetilde{\chi}_{1}^{0}$ & same as $\tilde{\chi}$ analyses \\
& $\widetilde{l}^{ \pm} \rightarrow \nu \widetilde{\chi}^{ \pm}$ & with $\leq 2$ extra leptons or $E$ \\
\hline
\end{tabular}

Table 3: Charged slepton and sneutrino pair production final states.

Sleptons may also decay directly to Standard Model particles via a $\lambda_{i j k}$ coupling. The possible decays then are: $\widetilde{l}_{L j} \rightarrow l_{k} \nu_{i}, \widetilde{l}_{L i} \rightarrow l_{k} \nu_{j}, \widetilde{l}_{R k} \rightarrow l_{j} \nu_{i}, l_{i} \nu_{j}$ (Fig. 3b). The topologies arising from sleptons decaying indirectly via the lightest neutralino $\widetilde{l} \rightarrow \widetilde{\chi}_{1}^{0} l$, consist of three charged leptons and missing energy (Fig. 3d). The latter decay is dominant in most of the MSSM parameter space. A mixed decay (one charged slepton decaying directly, the other one indirectly) is also possible. The final states resulting from sneutrino and slepton pair production are listed in Table 3.

Finally, the squarks cannot decay directly to Standard Model particles through an $L L \bar{E}$ operator; only the indirect decays via the lightest neutralino are possible $\left(\right.$ e.g $\left.\tilde{t} \rightarrow c \widetilde{\chi}_{1}^{0}, \tilde{b} \rightarrow b \tilde{\chi}_{1}^{0}\right)$.

\section{$1.4 \lambda_{i j k}$ couplings}

Upper limits on the $\lambda_{i j k}$ couplings can be derived from Standard Model processes $[8,14-$ 16], mainly charged-current universality, lepton universality, $\nu_{\mu}-\mathrm{e}$ scattering, forwardbackward asymmetry in $\mathrm{e}^{+} \mathrm{e}^{-}$collisions, and bounds on $\nu_{e}$-Majorana mass. Most present indirect limits on the $\lambda$ couplings derived from SM processes are in the range of $10^{-3}$ to $10^{-1}$; the most stringent upper limit is given for $\lambda_{133}$.

In case of pair production of supersymmetric particles, $R_{p}$ is conserved at the production vertex and the cross-section does not depend on the $R_{p}$ couplings. On the contrary, the $R_{p}$ decay width depends on the $\lambda$ strength, which then determines the mean decay length of the LSP. If the LSP is a neutralino or a chargino, the partial width is of a typical form for a fermion three-body decay [8] (see diagrams a and b in Fig. 2) and the mean decay length is given by $[17,18]$ :

$$
L(\mathrm{~cm})=0.3(\beta \gamma)\left(\frac{\mathrm{m}_{\tilde{\mathrm{l}}}}{100 \mathrm{GeV} / \mathrm{c}^{2}}\right)^{4}\left(\frac{1 \mathrm{GeV} / \mathrm{c}^{2}}{\mathrm{~m}_{\tilde{\chi}}}\right)^{5} \frac{1}{\lambda^{2}}
$$

where $\lambda=\lambda_{i j k}$ and $\beta \gamma=P_{\widetilde{\chi}} / m_{\tilde{\chi}}$. If the LSP is a sfermion, its $R_{p}$ decay is a two-body decay (see diagrams a and b in Fig. 3), and the mean decay length is given by:

$$
L(\mathrm{~cm})=10^{-12}(\beta \gamma)\left(\frac{1 \mathrm{GeV} / \mathrm{c}^{2}}{\mathrm{~m}_{\tilde{\mathrm{f}}}}\right) \frac{1}{\lambda^{2}}
$$

The analyses presented in this paper are valid if the $R$-parity violating decays are close to the production vertex, which means that the LSP flight path is shorter than a few 
centimeters. Considering the upper limits on the $\lambda_{i j k}$ derived from the Standard Model constraints and according to equation 1, the analyses are not sensitive in case of a light $\tilde{\chi}\left(M_{\chi_{L S P}} \leq 10 \mathrm{GeV} / c^{2}\right)$, due first to the term $m_{\tilde{\chi}^{-5}}$ and second to the term $(\beta \gamma)$ which becomes important. Moreover, when studying $\tilde{\chi}$ decays, for typical masses considered in this study, the analyses have a lower limit in sensitivity on the $\lambda$ coupling of the order of $10^{-4}$ to $10^{-5}$. In the opposite case, when the $R$-parity violating strength is very small, the LSP escapes the tracking devices before decaying and the results of the searches performed under the assumption of $R_{p}$ conservation are recovered [19]. To investigate intermediate coupling values, specific searches for displaced vertices should be performed.

For all the analyses presented in this paper, it was assumed that only one $\lambda_{i j k}$ is dominant at a time. Two kinds of searches have been performed:

- The first search assumes that $\lambda_{122}$ is dominant (i.e the charged leptons coming from $R_{p}$ decay are muons and electrons). In this case the neutralino decays into $\mathrm{e} \nu_{\mu} \mu$ or $\mu \nu_{e} \mu$ with a branching ratio of $50 \%$ for each channel. Then the corresponding final state for $\tilde{\chi}_{1}^{0}$ pair production is: missing energy, coming from the undetected neutrinos, and $2 \mathrm{e} 2 \mu(\approx 25 \%)$ or $1 \mathrm{e} 3 \mu(\approx 50 \%)$ or $4 \mu(\approx 25 \%)$. This is the most efficient case since the selection criteria depends on e and $\mu$ identification.

- The second search assumes that $\lambda_{133}$ is dominant, meaning that the leptons from $R_{p}$ decay are mainly taus, and electrons. This is the case with the lowest efficiency due to the presence of several taus in the final state.

The efficiencies for the other $\lambda_{i j k}$ lie between these two extreme cases. Two different $\lambda_{i j k}$ can lead to the same final state, and therefore the same efficiency ranges. For example, the results of the analysis designed for the $\widetilde{\nu}_{\mathrm{e}}$ direct decay via $\lambda_{133}$ can be applied to the $\widetilde{\nu}_{\mu}$ direct decay via $\lambda_{233}$ (see sections 3.2 and 4.2 ).

\section{Data samples}

The data corresponding to an integrated luminosity of $53 \mathrm{pb}^{-1}$ collected during 1997 by the DELPHI detector [20] at centre-of-mass energy around $183 \mathrm{GeV}$ were analysed. For the analyses depending on electron identification, an integrated luminosity of $50.7 \mathrm{pb}^{-1}$ was used after having imposed stringent data quality conditions on the barrel electromagnetic calorimeter.

Background contributions coming from the Standard Model processes $\mathrm{e}^{+} \mathrm{e}^{-} \rightarrow \mathrm{f} \overline{\mathrm{f}} \gamma, \gamma \gamma$, $\mathrm{e}^{+} \mathrm{e}^{-}, \mathrm{We} \nu_{\mathrm{e}}, \mathrm{Ze}^{+} \mathrm{e}^{-}, \mathrm{W}^{+} \mathrm{W}^{-}, \mathrm{ZZ}$ were considered. Event samples corresponding to the $\mathrm{q} \overline{\mathrm{q}}(\mathrm{n} \gamma), \tau^{+} \tau^{-}(\gamma)$ and $\mu^{+} \mu^{-}(\gamma)$ final states were produced by PYTHIA [21], KORALZ [22] and DYMU3 [23] respectively. Two-photon $(\gamma \gamma)$ interactions leading to leptonic final states were generated with the BDK program [24]; the reaction $\gamma \gamma \rightarrow$ hadrons was generated using TWOGAM [25]; biased samples containing events with a minimal transverse energy of $4 \mathrm{GeV}$ were used. For the study of four-fermion final states, the PYTHIA generator was used; a cross-check was performed using four-fermion final states generated with EXCALIBUR [26].

To evaluate signal efficiencies, sparticle production was generated using SUSYGEN [27]. Neutralino and chargino pair productions were considered at several points in the MSSM parameter space with different values of $\tan \beta$ (from 1 to 30 ), $m_{0}$ (between $5 \mathrm{GeV} / c^{2}$ and $500 \mathrm{GeV} / c^{2}$ ), $\mu$ (between $-200 \mathrm{GeV} / c^{2}$ and $200 \mathrm{GeV} / c^{2}$ ) and $M_{2}$ (between 5 and $400 \mathrm{GeV} / c^{2}$ ), for both $\lambda_{122}$ and $\lambda_{133}$ searches. To study sneutrino pair production, several signal configurations were generated: a $\tilde{\nu}$ mass range from 50 to $90 \mathrm{GeV} / c^{2}$ was covered, with $\lambda_{133}$ or $\lambda_{122}$, and with $\operatorname{Br}\left(\tilde{\nu} \rightarrow l^{+} l^{-}\right)=100 \%$. Events with sneutrino indirect decay were also simulated, for different $\tilde{\nu}$ and $\tilde{\chi}$ masses, in order to cover several ranges 
of mass difference between sneutrinos and neutralinos. The same type of procedure was applied to simulate charged slepton pair production and to study their direct and indirect decays. Finally, stop decays into a charm quark and a neutralino with the subsequent $R_{p}$ decay of the neutralino into leptons via a $\lambda_{i j k}$ coupling were also generated for several sets of stop and neutralino masses.

In the simulation of signal events, the $\lambda$ parameters were set to their present experimental upper limits: $\lambda_{122}=0.04$ and $\lambda_{133}=0.003$.

All generated signal events were processed with the DELPHI detector simulation program DELSIM.

\section{Analyses description}

Lepton identification. It is provided by different detector informations as described in [20]. Electron identification algorithm uses both the information from the $\mathrm{dE} / \mathrm{dX}$ measurement of the main tracking detector, the Time Projection Chamber, and the energy deposition in the electromagnetic calorimeters. Throughout this algorithm, three different levels of tagging are provided which classify electrons with momentum above $2 \mathrm{GeV} / c$. The analyses described in the following used the less severe level of tagging ("loose" tag) which give a typical efficiency of $80 \%$ with a probability of identifying a pion for an electron of $1.6 \%$. Muon identification algorithm relies on the association of charged particles with signals from the muon chambers, providing with four levels of tag. The analyses assuming that $\lambda_{122}$ is dominant used the two most severe levels ("standard" or "tight" tags) which have typical efficiencies of $86 \%$ and $76 \%$ respectively with a probability of identifying a pion as a muon of $0.7 \%$ and $0.4 \%$ respectively. The analyses concerning $\lambda_{133}$ coupling used a less severe level of tag ("loose" tag) which has a typical efficiency of $95 \%$ with a probability of identifying a pion as a muon of $1.5 \%$.

Jet reconstruction. As already mentioned, indirect decays of neutralinos or charginos can give two or more jets in the final state, beside leptons and missing energy. Moreover, in the case of the $\lambda_{133}$ coupling, the $\tau$ decays give isolated leptons or thin jets. The DURHAM [28] algorithm was used to reconstruct the jets. In order to cover the different topologies, the jet number was not fixed, and the jet charged multiplicity could be low (thin jets with one charged particle were possible), or could be zero in case of neutral jets. For each event, the DURHAM algorithm was applied to reconstruct from two to eight jets. The corresponding jet parameters were stored, in particular the transition value $\mathrm{Y}_{m n}$ of the $\mathrm{Y}_{\text {cut }}$ in the DURHAM algorithm at which the event changes from a $n$-jet to a $m$-jet configuration.

\subsection{Neutralinos and charginos decay}

The analyses described below were designed to cover all the final states listed in Table 2 as well as final states produced when the chargino is the LSP. Only the $2 l+\not E$ topology coming from direct chargino decays is not explicitly studied, since the region in the MSSM parameter space where this decay dominates is already covered by LEP1 model independent lower limit on the chargino mass $\left(\mathrm{m}_{\chi^{ \pm}}>45 \mathrm{GeV} / \mathrm{c}^{2}\right)[29]$.

\subsection{1 $\lambda_{122}$ case}

Events were selected if they satisfied the following criteria: 
- the charged multiplicity had to be greater than three, as the minimum number of charged particles expected in these topologies is four;

- the missing transverse momentum, $p_{t}$, was greater than $5 \mathrm{GeV} / c$ and the polar angle of the missing momentum was between $20^{\circ}$ and $160^{\circ}$.

This set of criteria reduced mainly the background coming from Bhabha scattering and two-photon processes. The following requirements were based on the lepton characteristics of the signal:

- at least two identified muons were asked;

- the energy of the most energetic identified lepton had to be greater than $10 \% \sqrt{s}$;

- an isolation criterion was imposed for the identified leptons (no other charged particle in a half cone of seven degrees around the lepton).

At this stage, most of the hadronic final states from $\mathrm{f} \overline{\mathrm{f}} \gamma, \mathrm{ZZ}$ and $\mathrm{W}^{+} \mathrm{W}^{-}$production were removed. The final criteria were designed to reduce contamination from the remaining semi-leptonic four-fermion final states:

- at least two of the identified leptons had to be leading particles in the jets;

- the polar angle of the jets in case of four, five, or six-jet topologies had to be between $20^{\circ}$ and $160^{\circ}$;

- the missing energy was at least $20 \% \sqrt{s}$.

At the end of the selection procedure, no event remained, while 0.7 events were expected from Standard Model processes, most of which came from $\mathrm{W}^{+} \mathrm{W}^{-}$(as shown in Table 5). For $\tilde{\chi}_{1}^{0} \tilde{\chi}_{1}^{0}$ the selection efficiencies were in the range $45-60 \%$, for $\tilde{\chi}_{1}^{+} \widetilde{\chi}_{1}^{-}: 20-50 \%$; and for $\tilde{\chi}_{2}^{0} \widetilde{\chi}_{1}^{0}: 25-40 \%$, for all the values of $\mu, M_{2}$ considered in this paper (see Table 5 ).

\subsection{2 $\lambda_{133}$ case}

The $\tau$ decay gives isolated leptons or thin jets and neutrinos. In this case the missing energy is expected to be greater than in the $\lambda_{122}$ case due to the presence of neutrinos, coming not only from neutralino or chargino $R_{p}$ decay, but also from $\tau$ decay.

Events were preselected if they satisfied the following criteria:

- at least one lepton was required;

- the number of charged particles had to be greater than three;

- the total energy and the energy from charged particles had to be greater than $0.18 \sqrt{s}$ and $0.16 \sqrt{s}$ respectively.

These above criteria removed around $99 \%$ of two-photon event samples used.

Several criteria were based on the missing quantities:

- the missing $p_{t}$ had to be greater than $5 \mathrm{GeV} / c$;

- the polar angle of the missing momentum had to be between $27^{\circ}$ and $153^{\circ}$;

- the missing energy had to be at least $0.30 \sqrt{s}$.

These requirements were efficient in suppressing the background coming from Bhabha, two-photon and $\mathrm{f} \bar{f} \gamma$ processes.

For events with fewer than eight charged particles, at least one lepton was required, whereas events with eight or more charged particles had to contain at least two leptons. In both cases, the energy of the most energetic lepton had to be greater than $5 \mathrm{GeV}$, and there should be no other charged particle in a $10^{\circ}$ half cone around the identified lepton(s). These criteria removed $\mathrm{ff} \gamma$ and hadronic $\mathrm{ZZ}$ and $\mathrm{W}^{+} \mathrm{W}^{-}$events. In Fig. 4 the distributions of the missing $p_{t}$, the energy of the most energetic lepton, and the minimum angle between the lepton and the nearest charged particle are presented. The agreement between real data and simulated background is fairly good. The distribution for simulated signal $\left(\widetilde{\chi}_{1}^{0} \widetilde{\chi}_{1}^{0}\right)$ is also plotted; it is scaled by a factor 10 in order to be visible. 


\begin{tabular}{|l|c|c|}
\hline \hline Selection criteria for $\lambda_{133}$ coupling & Data & MC \\
\hline At least one lepton & & \\
$N_{\text {charged }} \geq 4$ & & \\
$E_{\text {tot }} \geq 18 \% \sqrt{s}, E_{\text {charged }} \geq 16 \% \sqrt{s}$ & 1551 & $1479 \pm 13$ \\
Missing $p_{t}>5 \mathrm{GeV} / \mathrm{c}, 27^{\circ} \leq \Theta_{\text {miss }} \leq 153^{\circ}$ & 996 & $965 \pm 10$ \\
\hline$E_{\max }^{l} \geq 5 \mathrm{GeV}$ & 293 & $286 \pm 4$ \\
$\Theta_{\text {lepton-chargedparticle }}^{\min } \geq 10^{\circ}$ & 174 & $166 \pm 3$ \\
$E_{\text {miss }}>30 \% \sqrt{s}$ & 70 & $69.2 \pm 2.1$ \\
\hline If $N_{\text {charged }} \geq 8, N_{\text {lepton }} \geq 2$ & & \\
\hline \hline$E_{\text {cone }}^{30^{\circ}} \leq 50 \% E_{\text {total }}$ & 33 & $29.5 \pm 1.2$ \\
$Y_{34} \geq 0.001$ & 14 & $17.9 \pm 0.9$ \\
\hline In case of four or five jets, at least four charged jets & & \\
\hline Case of four jets: & & \\
$E_{\text {min }}^{j} * \theta_{\text {min }}^{j_{1}, j_{2}} \geq 0.5 \mathrm{GeV}$ rad, & & \\
$E_{\min }^{j} * \theta_{\min }^{j_{1}, j_{2}} \geq 5 \mathrm{GeV}$ rad if $N_{\text {charged }}>8$ & 3 & $3.3 \pm 0.3$ \\
$20^{\circ} \leq \theta_{\text {jet }} \leq 160^{\circ}$ & & \\
\hline
\end{tabular}

Table 4: List of selection criteria in searches for neutralino and chargino decays via the $\lambda_{133}$ coupling.

The final selection was based on the jet characteristics and topologies. First, the $\mathrm{Y}_{34}$ value had to be greater than $10^{-3}$, which reduced the $\mathrm{f} \overline{\mathrm{f}} \gamma$ contribution (Fig. 5). In case of four-jet or five-jet topologies, four charged jets were required. In case of a four jet topology, a cut was applied on the value of $E_{\min }^{j} \times \theta_{\min }^{j_{a} j_{b}}$ where $E_{\min }^{j}$ is the energy of the less energetic jet, and $\theta_{\text {min }}^{j j_{b}}$ is the minimum angle between any pair of jets. These requirements significantly reduced the background from $\mathrm{f} \overline{\mathrm{f}} \gamma, \gamma \gamma, \mathrm{W}^{+} \mathrm{W}^{-}$production. The number of remaining real and simulated data events after these criteria are reported in Table 4.

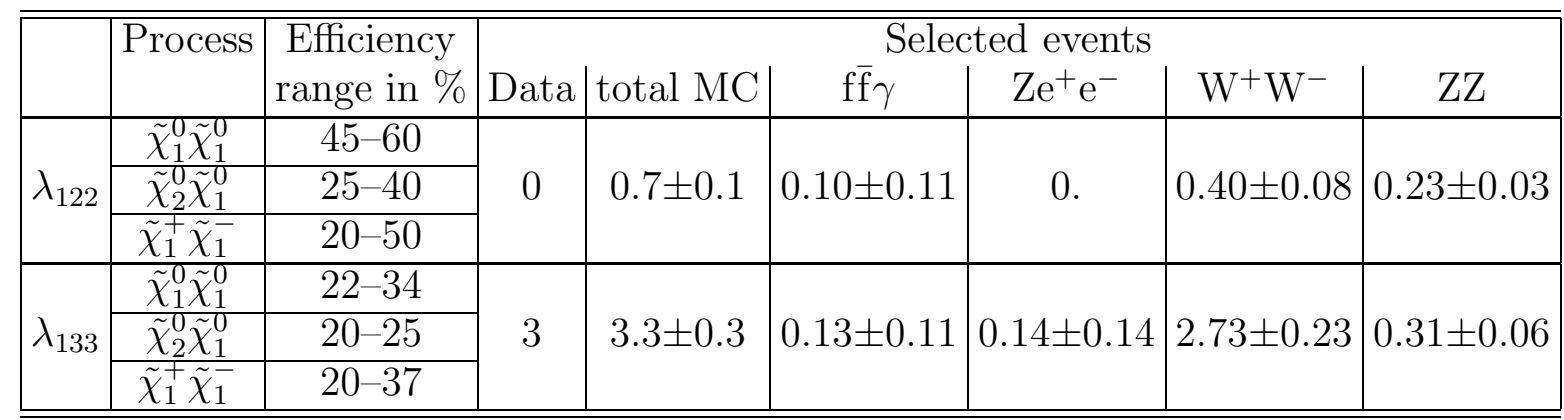

Table 5: Neutralino and chargino analyses: efficiency ranges for pair production processes, and data and Monte Carlo events selected for both $\lambda_{122}$ and $\lambda_{133}$. The neutralino and chargino mass ranges in the simulated signals are $15-50 \mathrm{GeV} / c^{2}$ and $45-90 \mathrm{GeV} / c^{2}$ respectively.

For $\tilde{\chi}_{1}^{0} \tilde{\chi}_{1}^{0}$ the selection efficiencies were in the range $22-34 \%$; for $\tilde{\chi}_{1}^{+} \widetilde{\chi}_{1}^{-}: 20-37 \%$; and for $\widetilde{\chi}_{2}^{0} \widetilde{\chi}_{1}^{0}: 20-25 \%$. Three events remained after the selection procedure with 3.3 expected from standard background processes, mainly from $\mathrm{W}^{+} \mathrm{W}^{-}$(Table 5).

The results obtained for both $\lambda_{122}$ and $\lambda_{133}$ couplings are summarized in Table 5 . 


\subsection{Sneutrinos decay}

The final state in $\tilde{\nu} \tilde{\bar{\nu}}$ production is typically purely leptonic. This is the case both for direct decay $(4 l)$ and for the dominant indirect decay via the lightest neutralino $(4 l+\not E)$. The latter decay is the dominant indirect mode since the results coming from chargino search (see Section 4.1) imply that the indirect decay to $\tilde{\chi}^{ \pm} l^{\mp}$ is negligible for a $\tilde{\nu}$ with a mass lower than $90 \mathrm{GeV} / c^{2}$.

In the case of the direct decay, the final states are $\mu \mu \mu \mu\left(\widetilde{\nu}_{\mathrm{e}}\right.$ pair $)$ or ee $\mu \mu\left(\widetilde{\nu}_{\mu}\right.$ pair $)$ with $\lambda_{122}$ and $\tau \tau \tau \tau\left(\widetilde{\nu}_{\text {e }}\right.$ pair $)$ or ee $\tau \tau\left(\widetilde{\nu}_{\tau}\right.$ pair $)$ with $\lambda_{133}$. The $4 \tau$ final state is also possible in the case of $\widetilde{\nu}_{\mu}$ pair decaying with a $\lambda_{233}$ coupling. In the case of the indirect decay in $\nu \widetilde{\chi}_{1}^{0}$, the same final states are obtained as for $\widetilde{\chi}_{1}^{0} \widetilde{\chi}_{1}^{0}$ production, with more missing energy. They depend on the $\lambda_{i j k}$ coupling, since the charged leptons are produced in the $\tilde{\chi}_{1}^{0} R_{p}$ decay. Therefore the efficiencies do not depend on the sneutrino type, but on the sneutrino and neutralino masses.

To be more efficient for all these purely leptonic final states, with at least four leptons, the selection criteria have been strengthened with respect to the chargino/neutralino analyses. In the case $\lambda_{122}$ dominates, the highest selection efficiency was obtained from the direct decays as described below; the indirect decays lead to final states already covered by the chargino and neutralino analysis described previously in section 3.1.1. In the case of the $\lambda_{133}$ coupling, the same analysis was used to study both direct and indirect decays since they lead to the same type of final state, only with some difference in missing energy.

\subsection{1 $\quad \lambda_{122}$ case}

If $\lambda_{122}$ is the dominant $R_{p}$ coupling, the direct decay mode leads to four leptons $(\mu$ or/and e) in the final state. The selection criteria are described below:

- the charged multiplicity had to be four;

- at least two muons were required;

- the total energy from charged particles had to be greater than $33 \% \sqrt{s}$;

- no other charged particle in a half cone of $10^{\circ}$ around the lepton, was demanded;

- the total event charge had to be 0 ;

- the missing energy had to be less than $55 \% \sqrt{s}$;

- the thrust value had to be less than 0.95 .

No event remained in the data after these criteria with 0.73 expected from standard background processes, mainly from the leptonic final states of the ZZ process (see Table 6). The efficiencies were evaluated by generating sneutrino pair production with masses from 50 to $90 \mathrm{GeV} / c^{2}$ and they were in the range $60-80 \%$ depending on the sneutrino mass (Table 7). In case of the sneutrino indirect decay, covered by the neutralino analysis, no detailed study has been performed to determine the efficiency as a function of $\mathrm{m}_{\tilde{\nu}}$ and $\mathrm{m}_{\widetilde{\chi}^{0}}$ since it has been done carefully in case of $\lambda_{133}$ coupling, which leads to the most conservative results.

\subsection{2 $\lambda_{133}$ case}

The preselection criteria were the same as in the case of chargino/neutralino studies, except that an upper limit of eight was set on the number of charged particles; the preselection eliminated more than $90 \%$ of all Standard Model backgrounds. A minimum requirement on the missing energy was imposed even in case of $4 l$ final states, due to the $\tau$ decay which produces a certain amount of missing energy. But compared to the 
neutralino decay in which neutrinos are produced directly, the missing energy is less important, therefore the limit was set to $0.1 \sqrt{s}$. The missing longitudinal momentum had to be less than $70 \mathrm{GeV} / c$.

The isolation criteria applied for the identified lepton(s) were also modified. If there were exactly four charged particles, the minimum angle between a lepton and the nearest charged particle had to be greater than $20^{\circ}$ otherwise it had to be greater than $6^{\circ}$.

As for the chargino/neutralino selection, several criteria were based on the jet characteristics. The DURHAM $Y_{34}$ and $\mathrm{Y}_{45}$ values (see Section 3.1) had to be greater than $1.8 \cdot 10^{-3}$ and $4 \cdot 10^{-4}$ respectively. In case of a four jet topology, there had to be no neutral jet, at least one jet with its leading particle identified as a lepton, and a minimum angle of $20^{\circ}$ between any pair of jets. The value of $E_{\min }^{j} \times \theta_{\min }^{j_{a} j_{b}}$ (see Section 3.1.2) had to be greater than $1 \mathrm{GeV}$ rad, and greater than $4 \mathrm{GeV}$ rad if the number of charged tracks was equal to eight.

One event remained after this selection with 1.8 expected from standard background processes. The background was mainly due to $\mathrm{W}^{+} \mathrm{W}^{-}, \gamma \gamma$ and $\mathrm{ZZ}$ production (Table 6). The selection efficiencies for both direct and indirect decays are summarized in Table 7 .

\begin{tabular}{|c|c|c|c|c|c|c|c|}
\hline Coupling & Data & $\mathrm{MC}$ & $\gamma \gamma$ & $\mathrm{ff} \gamma$ & $\mathrm{Ze}^{+} \mathrm{e}^{-}$ & $\mathrm{W}^{+} \mathrm{W}^{-}$ & $\mathrm{ZZ}$ \\
\hline$\lambda_{122}$ & 0 & $0.73 \pm 0.19$ & $0.14 \pm 0.01$ & $0.19 \pm 0.18$ & 0. & 0. & $0.40 \pm 0.06$ \\
\hline$\lambda_{133}$ & 1 & $1.81 \pm 0.28$ & $0.57 \pm 0.20$ & 0. & $0.14 \pm 0.14$ & $0.67 \pm 0.11$ & $0.42 \pm 0.07$ \\
\hline
\end{tabular}

Table 6: Background contributions from standard model processes in the sneutrino analysis.

\begin{tabular}{|c|l|l|c|c|c|}
\hline Coupling & Decay & Characteristics & \multicolumn{2}{|c|}{ Efficiency } & \multicolumn{2}{|c|}{ Selected events } \\
& & & range in \% & Data & MC \\
\hline \hline$\lambda_{122}$ & $\widetilde{\nu}_{\mathrm{e}} \rightarrow \mu^{+} \mu^{-}$ & Direct decay & $60-80$ & 0 & $0.8 \pm 0.1$ \\
& $\widetilde{\nu}_{\mu} \rightarrow \mathrm{e}^{ \pm} \mu^{\mp}$ & Direct decay & $50-70$ & & \\
\hline \hline \multirow{4}{*}{$\lambda_{133}$} & $\widetilde{\nu}_{\mathrm{e}} \rightarrow \tau^{+} \tau^{-}$ & Direct decay & $32-37$ & & \\
& $\widetilde{\nu}_{\tau} \rightarrow \mathrm{e}^{ \pm} \tau^{\mp}$ & Direct decay & $41-47$ & & \\
\cline { 2 - 4 } & \multirow{2}{*}{} & $20<\widetilde{\chi}_{1}^{0}$ mass $<30$ & $18-29$ & \multirow{3}{*}{$1.8 \pm 0.2$} \\
& \multirow{\nu}{*}{$\widetilde{\chi}_{1}^{0} \nu$} & $30<\widetilde{\chi}_{1}^{0}$ mass $<40$ & $27-36$ & & \\
& & $\widetilde{\chi}_{1}^{0}$ mass $>40$ & $35-39$ & & \\
\hline
\end{tabular}

Table 7: Sneutrino analysis: efficiency ranges in the different cases studied, and data and Monte Carlo events remaining after the applied selection. Sneutrinos were generated with masses in the range $50-90 \mathrm{GeV} / c^{2}$.

\subsection{Charged sleptons decay}

Right-handed sleptons ${ }^{1}$ have been studied here, because their production cross-section is lower than for the left-handed ones, therefore leading to more conservative results. A particular analysis is devoted to the search for the direct decay of the slepton pair, leading to the $2 l+E$ final state. According to our present limit on the chargino mass (see Section 4.1), the branching fraction of the indirect slepton decay into $\nu \widetilde{\chi}^{ \pm}$is negligible; the dominant indirect decay into $l \widetilde{\chi}_{1}^{0}$ gives mostly purely leptonic final states, with at least six charged leptons. The selection efficiencies depend on the slepton and neutralino

\footnotetext{
${ }^{1}$ In this section the term "slepton" means charged slepton
} 
masses, but contrary to the $\tilde{\nu}$ case, for any given type of coupling, they also depend on the slepton family since there are always two leptons of the same flavour in the final state.

\section{- Analysis of direct slepton decays}

With $\lambda_{133}$ coupling, among the right-handed sleptons, only the $\tilde{\tau}_{R}$ can decay directly, and it has two decay modes: $\tilde{\tau} \rightarrow \tau \nu_{e}(50 \%), \tilde{\tau} \rightarrow$ e $\nu_{\tau}(50 \%)$. Then the final state in pair production of $\tilde{\tau}$ is: missing energy + ee $(25 \%)$, e $\tau(50 \%), \tau \tau(25 \%)$. Three specific analyses were performed for the three components of the final state. Several preselection criteria were common to the ee $+E$ and $\mathrm{e} \tau+\not E$ analyses:

- the missing $p_{t}$ had to be greater than $20 \mathrm{GeV} / c$ and the polar angle of the missing momentum was required to lie between $25^{\circ}$ and $155^{\circ}$;

- the acollinearity had to be greater than $10^{\circ}$ and the acoplanarity less than $160^{\circ}$;

- the energy of the most energetic photon was required to be less than $10 \mathrm{GeV}$.

Then different criteria were applied to discriminate between the two channels:

ee $+E$ final state

- two electrons were required; the angle between them had to be at least $10^{\circ}$ and at most $160^{\circ}$;

- the energy of each electron had to be greater than $10 \mathrm{GeV}$, and the sum of their energies less than $110 \mathrm{GeV}$;

- the neutral multiplicity of the event had to be less than 2 .

After the above selection, no event remained in the data with 1.3 expected from standard background processes (see Table 8).

$\mathbf{e} \tau+\not E$ final state

- the charged multiplicity and the neutral multiplicity had to be both less than 5 ;

- at least one electron was required, and not more than one muon;

- if one muon was tagged, the charged multiplicity had to be two (one $\mu$, one e);

- the total event charge had to be 0 ;

- the total energy in charged particles had to be greater than $5 \% \sqrt{s}$ and lower than $65 \% \sqrt{s}$

- the minimum angle between the lepton and the closest charged particle had to be at least $10^{\circ}$, at most $160^{\circ}$, and the minimum angle between the lepton and the nearest neutral had to be greater than $10^{\circ}$;

- the total electromagnetic energy had to be at least $10 \mathrm{GeV}$, and the total leptonic energy had to be between 10 and $110 \mathrm{GeV}$

After applying the above criteria one event remained in the data with 2.8 expected from standard background processes (Table 8).

\begin{tabular}{|c|c|c|c|c|c|c|c|c|c|}
\hline \multirow[t]{2}{*}{ Channel } & \multicolumn{7}{|c|}{$\begin{array}{l}\text { Efficiencies (\%) as function } \\
\text { of } \tilde{\tau} \text { mass }\left(\mathrm{GeV} / c^{2}\right)\end{array}$} & \multicolumn{2}{|c|}{ Selected events } \\
\hline & 50 & 55 & 60 & 65 & 70 & 75 & 80 & Data & Background \\
\hline & 30 & 32 & 35 & 33 & 35 & 41 & 40 & 0 & $1.3 \pm 0.1$ \\
\hline e $\tau+E$ & 19 & 21 & 22 & 22 & 22 & 27 & 29 & 1 & $2.8 \pm 0.2$ \\
\hline
\end{tabular}

Table 8: Slepton direct decay: efficiencies for several values of $\tilde{\tau}$ masses and data and Monte Carlo events remaining after the applied selection, for both channels.

For the $\tau \tau+\mathbb{E}$ final state the analysis performed for the search of $R_{p}$ conserved $\tilde{\tau} \rightarrow \tau \widetilde{\chi}_{1}^{0}$ decay [30] have been used: 7 events were selected for 7.5 expected, with an efficiency of $31 \%$ which was rather stable in the $\tilde{\tau}$ mass range considered. 


\section{- Analysis of indirect slepton decays}

In the case of the $\lambda_{122}$ analysis, the case of maximum efficiency was studied, namely the indirect smuon decay; the selection criteria consisted of:

- charged multiplicity greater than or equal to four,

- at least three muons were required,

- the total leptonic energy greater than $80 \mathrm{GeV}$.

In the case of the $\lambda_{133}$ analysis, the same criteria were used as for the sneutrino searches. Efficiencies and results are reported in Table 9.

\begin{tabular}{|c|l|c|c|c|c|}
\hline Coupling & Decay & $\tilde{\chi}_{1}^{0}$ mass range & Efficiency & \multicolumn{2}{|c|}{ Selected events } \\
& & in GeV $/ c^{2}$ & range in \% & Data & MC \\
\hline \hline$\lambda_{122}$ & $\tilde{\mu} \rightarrow \mu \widetilde{\chi}_{1}^{0}$ & $50-80$ & $70-80$ & 0 & $0.3 \pm 0.1$ \\
\hline \hline \multirow{3}{*}{$\lambda_{133}$} & $\tilde{\mathrm{e}} \rightarrow \mathrm{e} \widetilde{\chi}_{1}^{0}$ & $50-80$ & $35-39$ & & \\
\cline { 2 - 4 } & $\tilde{\mu} \rightarrow \mu \widetilde{\chi}_{1}^{0}$ & $50-80$ & $42-48$ & & \\
\cline { 2 - 4 } & \multirow{2}{*}{$\rightarrow \tau \widetilde{\chi}_{1}^{0}$} & $25-35$ & $24-29$ & \multirow{2}{*}{$1.8 \pm 0.3$} \\
\cline { 3 - 4 } & & $35-45$ & $25-32$ & & \\
\cline { 3 - 4 } & & $45-80$ & $26-34$ & & \\
\hline
\end{tabular}

Table 9: Slepton analyses: efficiency ranges in the different cases studied and data and Monte Carlo events remaining after the applied selection. Sleptons were generated with masses in the range $50-90 \mathrm{GeV} / c^{2}$.

\subsection{Stop indirect decay}

With a $\lambda$ coupling, only the indirect decay of a squark into a quark and a neutralino (or a chargino) is possible. In the case of stop pair production, each of the stops decays into a charm quark and a neutralino, giving two jets + four charged leptons + missing energy in the final state. This signature is similar to the one produced by the indirect decay of the heavier neutralino into $\widetilde{\chi}_{1}^{0}$ and $\mathrm{Z}^{*}$, with one of the $\mathrm{Z}$ giving two jets, and the other giving two neutrinos. Therefore the analysis used in neutralino and chargino searches (see 3.1) was also used in this case. The highest efficiency was obtained when the dominant coupling is $\lambda_{122}$; in this case, the same analysis was used as for the neutralino and chargino decay study (see Section 3.1.1), giving an efficiency of $34 \%$ for $\mathrm{m}_{\tilde{t}}=70 \mathrm{GeV} / \mathrm{c}^{2}$ and $\mathrm{m}_{\widetilde{\chi}^{0}}=50 \mathrm{GeV} / c^{2}$. A more detailed study was performed to determine efficiencies in the case of a dominant $\lambda_{133}$ coupling, since it leads to the most conservative limit on the stop mass. The same selection criteria as described in Section 3.1.2 were used, but since in the case of stop pair production the final state always contains two jets, a minimum multiplicity of eight charged particles was required. The distributions of the number of identified leptons, of the missing energy and of the product $E_{\min }^{j} \times \theta_{\min }^{j_{1}, j_{2}}$ versus the number of charged particles obtained after preselection criteria are shown on Fig. 6 .

At least two identified leptons were required, and in the case of two or three identified leptons, there had to be no other charged particle in a $10^{\circ}$ half cone around them. The final criteria based on the jet characteristics and topologies were slightly modified: first, $\log _{10}\left(Y_{34}\right)$ had to be greater than -2.5 , and second, in case of a four jet topology, four charged jets were required and the value of $E_{\min }^{j} \times \theta_{\text {min }}^{j a j_{b}}$ had to be greater than $5 \mathrm{GeV}$ rad. Three events remained after the selection procedure, with 4.9 expected from background contribution (see Table 10). 


\begin{tabular}{|l|c|c|}
\hline \hline Selection criteria & Data & MC \\
\hline at least one lepton & & \\
$N_{\text {charged }} \geq 8$ & & \\
$E_{\text {charged }} \geq 18 \% \sqrt{s}, E_{\text {tot }} \geq 16 \% \sqrt{s}$ & & \\
missing $p_{t}>5 \mathrm{GeV} / c$ & & \\
$27^{\circ} \leq \Theta_{\text {miss }} \leq 153^{\circ}$ & 508 & $453 \pm 7$ \\
$E_{\operatorname{miss}}>30 \% \sqrt{s}$ & 347 & $315 \pm 6$ \\
\hline$E_{\max }^{l} \geq 5 \mathrm{GeV}$ & 125 & $116 \pm 2$ \\
\hline$\Theta_{\text {lepton-chargedparticle }}^{\min } \geq 10^{\circ}$ if $N_{\text {lepton }} \leq 3$ & 21 & $19.7 \pm 1.3$ \\
\hline$N_{\text {lepton }} \geq 2$ & 18 & $17.5 \pm 1.2$ \\
\hline \hline $\log _{10}\left(Y_{34}\right) \geq-2.5$ & & \\
\hline case of four jets : & & \\
at least four charged jets & 3 & $4.9 \pm 0.5$ \\
$E_{\min }^{j} * \theta_{\text {min }}^{j_{1}, j_{2}} \geq 5 \mathrm{GeV} . \mathrm{rad}$ & & \\
$20^{\circ} \leq \theta_{\text {jet }} \leq 160^{\circ}$ & & \\
\hline
\end{tabular}

Table 10: Selection criteria for the stop indirect decay analysis.

Selection efficiencies varied with the stop mass and with the mass difference between the stop and the lightest neutralino. If this mass difference was higher than $5 \mathrm{GeV} / c^{2}$, the efficiency lay between 21 and $29 \%$. In the degenerate case (i.e the mass difference around $5 \mathrm{GeV} / \mathrm{c}^{2}$ ), the efficiency decreased and lay between 15 and $19 \%$. This analysis was not optimized for topologies produced when the mass difference is below $4 \mathrm{GeV} / c^{2}$, therefore it was not sensitive to the very degenerate case.

\section{Interpretation of $\lambda$ dominant searches in terms of MSSM parameters}

By performing the analyses described in the previous sections at $\sqrt{s}=183 \mathrm{GeV}$, no excess of events was found in the data with respect to the Standard Model expectation. As a consequence, limits on the production cross-section and the mass of the sparticles was set. Similar searches performed by the other three LEP experiments have also shown no evidence for $R_{p}$ violating effects [31].

\subsection{Results from neutralino and chargino studies}

Both direct and indirect decays of pair production of charginos and neutralinos were combined to give the exclusion contours at $95 \%$ C.L. in the $\mu, M_{2}$ plane. For each coupling, the selection criteria were sensitive to most of the possible decay channels of neutralinos and charginos produced in the three processes considered $\left(\tilde{\chi}_{1}^{0} \tilde{\chi}_{1}^{0}, \tilde{\chi}_{2}^{0} \tilde{\chi}_{1}^{0}, \tilde{\chi}_{1}^{+} \tilde{\chi}_{1}^{-}\right)$. The number of expected events $N_{\text {exp }}$, for a given set of MSSM parameters is:

$$
N_{\text {exp }}=\mathcal{L} \times \sum_{i=1}^{i=3} \epsilon_{i} \sigma_{i}
$$

where $\epsilon_{i}$ gives the efficiency for each process, $\sigma_{i}$ the corresponding cross-section and $\mathcal{L}$ the integrated luminosity. The number of signal events, $N_{95}$, expected at the $95 \%$ confidence level in the presence of background is given by the standard formula [29]. All the points in 
the $\mu, M_{2}$ plane which satisfied the condition $N_{\exp }>N_{95}$ were excluded at $95 \%$ C.L. The exclusion contours for two values of $\tan \beta$ and $m_{0}$ are shown on Fig. 7. The light grey area shows the region excluded by the $\lambda_{133}$ search and the dark grey area the additional region excluded by the $\lambda_{122}$ search which, having a better efficiency, includes and extends the $\lambda_{133}$ region. One can consider these two searches as the most and the least sensitive cases. The other couplings have a sensitivity lying in between these two extremes, therefore the reported results are valid for any choice of $\lambda$ coupling.

By considering the $\lambda_{133}$ excluded area in the parameter space, (i.e the most conservative case), when scanning over $m_{0}$ for several values of $\tan \beta$, a lower limit on neutralino mass was obtained. For small values of $m_{0}$, the pair production of charginos is suppressed due to the destructive interference between the $s$ and $t$-channel, but the neutralino cross-section is enhanced due to the $t$-channel $\tilde{\mathrm{e}}$ exchange. Contrary to the $R$-parity conservation scenario, as already explained, $\widetilde{\chi}_{1}^{0} \widetilde{\chi}_{1}^{0}$ and $\widetilde{\chi}_{1}^{0} \widetilde{\chi}_{2}^{0}$ lead to visible final states, allowing to exclude such regions. For higher values of $m_{0}$, the chargino pair production dominates in most of the parameter space. For any given $\tan \beta$, the most conservative limit on the neutralino mass was obtained from this region, i.e high values of $m_{0}\left(\geq 500 \mathrm{GeV} / c^{2}\right)$. The result of the scan is shown in Fig. 8, which gives the neutralino mass limit as a function of $\tan \beta$, independently of the choice of $m_{0}$.

The same procedure has been applied to determine the most conservative lower limit on the chargino mass. The result is less dependent on $\tan \beta$, allowing to almost reach the kinematical limit for any value of $\tan \beta$. In summary out of this search, neutralinos with mass less than $27 \mathrm{GeV} / c^{2}$ and charginos with mass less than $89 \mathrm{GeV} / c^{2}$ were excluded at $95 \%$ C.L.

\subsection{Results from sneutrino studies}

The pair production of sneutrinos decaying either directly into two charged leptons each, or indirectly into a neutralino and a neutrino was studied.

The efficiencies obtained from the $4 l$ channels, for different values of the sneutrino mass, combined with the results of the selection on data and background, allowed the derivation of a limit on the cross-section as a function of the $\tilde{\nu}$ mass, shown on Fig. 9. On the same plot the MSSM cross-sections of $\mathrm{e}^{+} \mathrm{e}^{-} \rightarrow \tilde{\nu} \tilde{\bar{\nu}}$ versus the $\tilde{\nu}$ mass are shown. The pair production cross-section of $\widetilde{\nu}_{\mu}$ and $\widetilde{\nu}_{\tau}$ depends only on the $\tilde{\nu}$ mass, whereas in the $\widetilde{\nu}_{\mathrm{e}}$ case there is a strong dependence on the chargino mass and field component, due to the contribution of the t-channel chargino exchange. For chargino masses greater than $\approx$ $400 \mathrm{GeV} / c^{2}$, the production cross sections of all three sneutrino families tend to be equal $\sigma\left(\mathrm{e}^{+} \mathrm{e}^{-} \rightarrow \widetilde{\nu}_{\mathrm{e}} \widetilde{\bar{\nu}}_{\mathrm{e}}\right)=\sigma\left(\mathrm{e}^{+} \mathrm{e}^{-} \rightarrow \widetilde{\nu}_{\mu} \widetilde{\bar{\nu}}_{\mu}\right)$. The dashed upper curve of the same plot shows the $\widetilde{\nu}_{\mathrm{e}}$ cross-section obtained for $\mu=-200 \mathrm{GeV} / c^{2}$ and $M_{2}=100 \mathrm{GeV} / c^{2}$, in which the corresponding chargino mass lies between 90 and $120 \mathrm{GeV} / c^{2}$. From the direct topologies the most conservative limit on the sneutrino mass was derived at $63 \mathrm{GeV} / c^{2}$, as can be seen on the same figure.

The indirect decays of the sneutrinos lead to the same signature as the neutralino $R_{p}$ decay with additional missing energy. As in the chargino/neutralino study, the most conservative limit was obtained from the $\lambda_{133}$ coupling. Taking into account the efficiencies obtained as a function of sneutrino and neutralino masses and the analysis results, the $95 \%$ C.L exclusion region was derived in the $\mathrm{m}_{\tilde{\nu}}, \mathrm{m}_{\tilde{\chi}_{1}^{0}}$ plane (Fig. 10). The largest exclusion domain was obtained from $\mathrm{e}^{+} \mathrm{e}^{-} \rightarrow \widetilde{\nu}_{\mathrm{e}} \widetilde{\bar{\nu}}_{\mathrm{e}}$ process, at values of $\mu$ and $M_{2}$ equal to $-200 \mathrm{GeV} / c^{2}$ and $100 \mathrm{GeV} / c^{2}$ respectively and a chargino mass close to the kinematic limit. The smallest exclusion area was obtained from $\widetilde{\nu}_{\mu} \widetilde{\bar{\nu}}_{\mu}, \widetilde{\nu}_{\tau} \widetilde{\bar{\nu}}_{\tau}$ process, but is also valid 
for $\widetilde{\nu}_{\mathrm{e}} \widetilde{\bar{\nu}}_{\mathrm{e}}$ production in case of a heavy chargino. The reduction of the exclusion domain for low values of neutralino mass is due to the decrease of the selection efficiencies at this area. Considering the sneutrino indirect decays and when taking into account the limit on the neutralino mass at $27 \mathrm{GeV} / c^{2}$, sneutrinos with mass lower than $62 \mathrm{GeV} / c^{2}$ were excluded at $95 \%$ C.L.

In the same figure the limits obtained in the case of direct $\tilde{\nu}$ decay are shown. The line labelled $\lambda_{233}$ corresponds to $\widetilde{\nu}_{\mu}$ pair production leading to a $4 \tau$ final state. This limit is lower than the one obtained for the indirect decay into $\widetilde{\chi}_{1}^{0} \nu$ via a $\lambda_{133}$ coupling when $\mathrm{m}_{\tilde{\chi}^{0}}$ is greater than $30 \mathrm{GeV} / c^{2}$, since in this case the final state is a mixing of $4 \tau, 1 \mathrm{e} 3 \tau, 2 \mathrm{e} 2 \tau$, and the efficiency is slightly higher.

In summary, the most conservative limit from the sneutrino searches was derived by the indirect topologies leading to a lower bound on the sneutrino mass at $62 \mathrm{GeV} / c^{2}$.

\subsection{Results from charged slepton studies}

To obtain conservative limits, the pair production of right-handed sleptons was studied, since its cross-section is smaller than the left-handed one for a given slepton mass. Both direct decays of sleptons to charged sleptons and neutrinos and indirect to neutralinos and charged leptons were considered.

For the direct decay searches, the results obtained from the three analyses described in Section 3.3 were combined and limits on the production cross-section as a function of slepton mass were derived at $95 \%$ C.L (Fig. 11). The figure also shows the MSSM $\widetilde{l}_{R} \widetilde{l}_{R}$ production cross-sections. A lower limit on the slepton mass was set at $61 \mathrm{GeV} / c^{2}$.

For the indirect decay searches, the most conservative limit was obtained considering the $\lambda_{133}$ coupling as stated before. From the results of the analyses described in section 3.3, an exclusion region was derived in the $\mathrm{m}_{\tilde{l}}, \mathrm{~m}_{\tilde{\chi}_{1}^{0}}$ plane (Fig. 12). As direct topologies lead to poorer limits on slepton masses due to the higher remaining background, our present lower limit on slepton mass was set by these studies at $61 \mathrm{GeV} / c^{2}$ at $95 \%$ C.L.

\subsection{Results from stop studies}

From the study of the stop indirect decay to charm and neutralino, with the subsequent $R_{p}$ decay of the neutralino in leptons, a lower limit on the stop pair production crosssection was derived as a function of the stop and neutralino masses. Using the efficiencies determined for various values of the neutralino mass, and considering the lowest MSSM cross-section for the stop pair production in case of a maximal decoupling to the $\mathrm{Z}$ boson (mixing angle $=56^{\circ}$ ), the exclusion limit was derived in the $\mathrm{m}_{\tilde{t}}, \mathrm{~m}_{\tilde{\chi}_{1}^{0}}$ plane, as shown in Fig. 13. Taking into account our result on the neutralino mass limit of $27 \mathrm{GeV} / c^{2}$ the lower bound on stop mass is $61 \mathrm{GeV} / c^{2}$ at $95 \%$ C.L., valid for a mass difference between the stop and the neutralino greater than $4 \mathrm{GeV} / c^{2}$.

\section{Conclusion}

Searches for $R_{p}$ effects in $\mathrm{e}^{+} \mathrm{e}^{-}$collisions at $\sqrt{s}=183 \mathrm{GeV}$ have been performed with the DELPHI detector. The pair production of supersymmetric particles has been studied for the $\lambda$ type of $R_{p}$ operators assuming that the LSP has a negligible lifetime and that the $\lambda$ couplings are strong enough for the LSP to decay inside the detector. No evidence for $R$-parity violation has been observed, allowing the exclusion of a large domain of MSSM 
parameters. In all cases the most conservative limit has been derived which is valid for all the generation indices $i, j, k$ of the $\lambda_{i j k}$ coupling.

From the study of the neutralino and chargino direct and indirect decays, a limit on the mass of the lightest neutralino of $27 \mathrm{GeV} / c^{2}$ has been deduced. This limit was set independently of the choice of $m_{0}$. Furthermore a chargino with mass lighter than $89 \mathrm{GeV} / c^{2}$ has been excluded at $95 \%$ confidence level.

Studies of both direct and indirect decays of charged sleptons and sneutrinos have been performed. The most conservative mass limit of $61 \mathrm{GeV} / c^{2}$ on the charged sleptons has been obtained by the search for their direct $R_{p}$ decay, as opposed to the sneutrino case in which the most conservative result was obtained for the indirect $R_{p}$ decays and led to a lower mass limit of $62 \mathrm{GeV} / c^{2}$.

Finally, searches for indirect stop decay into a charm quark and a neutralino and the subsequent decay of the neutralino via $\lambda$ couplings, led to a limit on the squark mass of $61 \mathrm{GeV} / c^{2}$ at $95 \%$ confidence level.

\section{Acknowledgements}

We are greatly indebted to our technical collaborators, to the members of the CERNSL Division for the excellent performance of the LEP collider, and to the funding agencies for their support in building and operating the DELPHI detector.

We acknowledge in particular the support of Austrian Federal Ministry of Science and Traffics, GZ 616.364/2-III/2a/98, FNRS-FWO, Belgium,

FINEP, CNPq, CAPES, FUJB and FAPERJ, Brazil, Czech Ministry of Industry and Trade, GA CR 202/96/0450 and GA AVCR A1010521, Danish Natural Research Council,

Commission of the European Communities (DG XII),

Direction des Sciences de la Matière, CEA, France,

Bundesministerium für Bildung, Wissenschaft, Forschung und Technologie, Germany,

General Secretariat for Research and Technology, Greece,

National Science Foundation (NWO) and Foundation for Research on Matter (FOM), The Netherlands,

Norwegian Research Council,

State Committee for Scientific Research, Poland, 2P03B06015, 2P03B03311 and SPUB/P03/178/98,

JNICT-Junta Nacional de Investigação Científica e Tecnológica, Portugal,

Vedecka grantova agentura MS SR, Slovakia, Nr. 95/5195/134,

Ministry of Science and Technology of the Republic of Slovenia,

CICYT, Spain, AEN96-1661 and AEN96-1681,

The Swedish Natural Science Research Council,

Particle Physics and Astronomy Research Council, UK,

Department of Energy, USA, DE-FG02-94ER40817. 


\section{References}

[1] H.P. Nilles, Phys. Rep. 110 (1984) 1.

[2] H.E. Haber and G.L. Kane, Phys. Rep. 117 (1985) 75.

[3] S. P. Martin, in Perspectives on supersymmetry, edited by G.L. Kane, 1-98; hep-ph/9709356

[4] P. Fayet, Phys. Lett. B69 (1977) 489; G. Farrar and P. Fayet, Phys. Lett. B76 (1978) 575.

[5] S. Weinberg, Phys. Rev. D26 (1982) 287.

[6] I. Hinchliffe and T. Kaeding, Phys. Rev. D47 (1993) 279.

[7] C.E. Carlson et al., Phys. Lett. B357 (1995) 99.

[8] V. Barger, G.F. Guidice and T. Han, Phys. Rev. D40 (1989) 2987.

[9] J. Ellis and S. Rudaz, Phys. Lett. B128 (1983) 248.

[10] A. Bartl et al., Zeit. Phys. C76 (1997) 549.

[11] M. Drees, "An introduction to supersymmetry" Lectures given at Inauguration Conference of the Asia Pacific Center for Theoretical Physics (APCTP), Seoul, Korea, 4-19 Jun 1996; hep-ph/9611409

[12] M. Drees and S.P. Martin, "Implications of SUSY model building," hep-ph/9504324.

[13] M. Drees and K. Hikasa, Phys. Lett. B252 (1990) 127.

[14] H. Dreiner, hep-ph/9707435.

[15] G. Bhattacharyya, hep-ph/9709395 and Nucl. Phys.B (Proc. Suppl.) 52A (1997) 83.

[16] R. Barbier et al., Report of the group on the R-parity violation, hep-ph/9810232.

[17] S. Dawson, Nucl. Phys. B261 (1985) 297.

[18] H. Dreiner and G.G. Ross, Nucl. Phys. B365 (1991) 597.

[19] DELPHI Collaboration, CERN-EP/98-171. DELPHI Collaboration, CERN-EP/98-176.

[20] P. Abreu et al., Nucl. Instr. Meth. 378 (1996) 57.

[21] T. Sjostrand, Computer Phys. Comm. 39 (1986) 347.

[22] S. Jadach and Z. Was, Computer Phys. Comm. 79 (1994) 503.

[23] J.E. Campagne and R. Zitoun, Zeit. Phys. C43 (1989) 469.

[24] F.A. Berends, P.H. Davervelt and R. Kleiss, Computer Phys. Comm. 40 (1986) 271,285 and 309.

[25] S. Nova, A. Olshevski and T. Todorov, DELPHI 90-35 PROG 152.

[26] F.A. Berends, R. Kleiss and R. Pittau, Computer Phys. Comm. 85 (1995) 437.

[27] S. Katsanevas and P. Morawitz, hep-ph/9711417, submitted to Comp. Phys. Comm.

[28] S. Catani et al., Phys. Lett. B269 (1991) 432.

[29] Particle Data Group, Phys. Rev. D54 (1996) 1.

[30] M. Berggren, M. Gandelman and J.H.Lopes, DELPHI 97-156 PHYS 735.

[31] ALEPH Collaboration (R. Barate et al.) Eur. Phys. J. C4 (1998) 433.

Contributions for ICHEP'98 (preliminary results):

ALEPH Collaboration, ALEPH 98-070 CONF 98-039;

DELPHI Collaboration, DELPHI 98-138 CONF 199;

L3 Collaboration, L3 Note 229;1

OPAL Collaboration, Physics Notes 356, 359. 

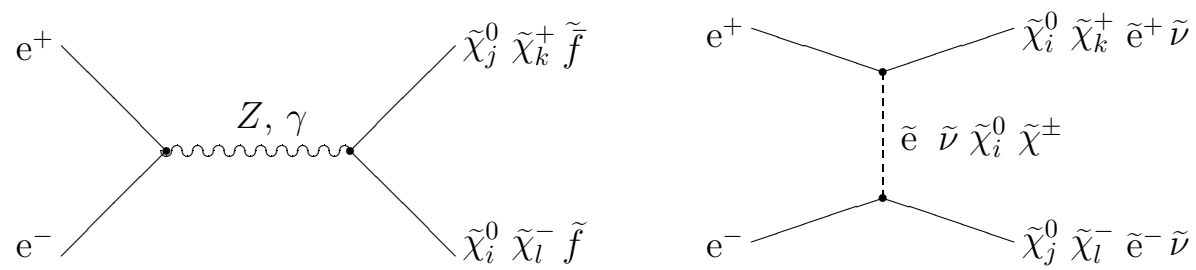

Figure 1: Neutralino, chargino and sfermion pair production diagrams $(i, j=1, . .4$; $k, l=1,2)$. In the $s$-channel neutralinos and neutral sfermions are produced only via the $\mathrm{Z}$.
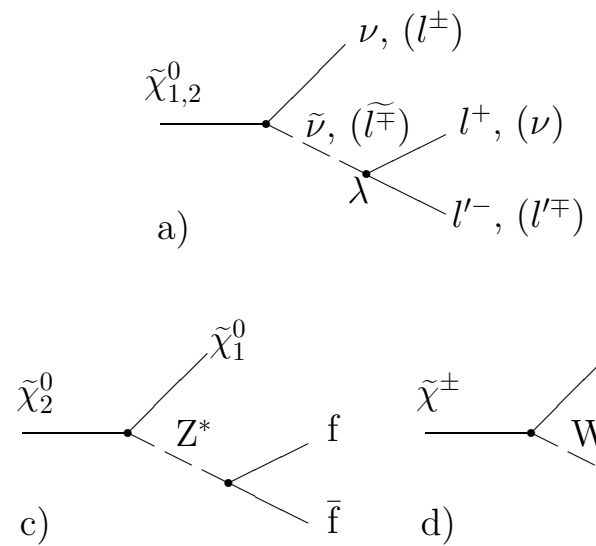

c)

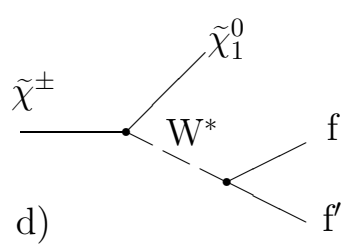

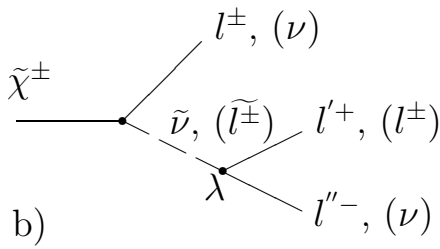

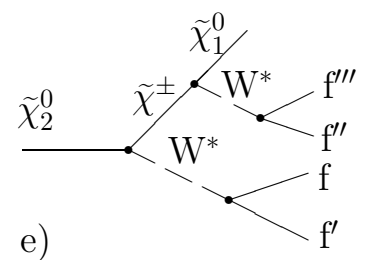

Figure 2: Neutralino and chargino decay diagrams. a: $\widetilde{\chi}^{0}$ direct decay; b: $\widetilde{\chi}^{ \pm}$direct decay; in these diagrams the $\lambda$ indicates the $R_{p}$ vertex. c, e: $\widetilde{\chi}_{2}^{0}$ indirect decay; d: $\widetilde{\chi}^{ \pm}$indirect decay; the subsequent neutralino $R_{p}$ decay is shown in a.

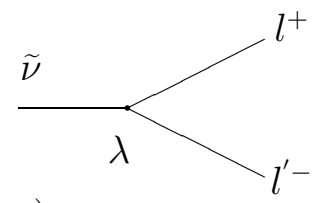

a)

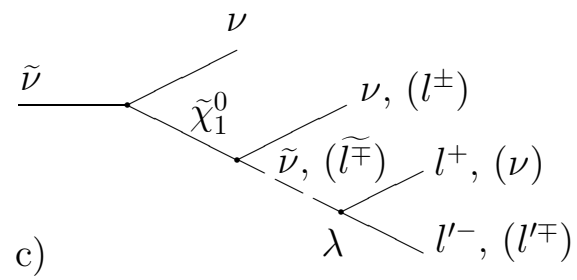

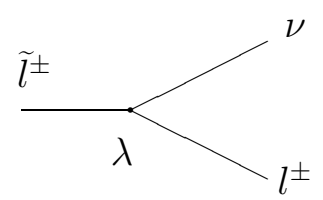

b)

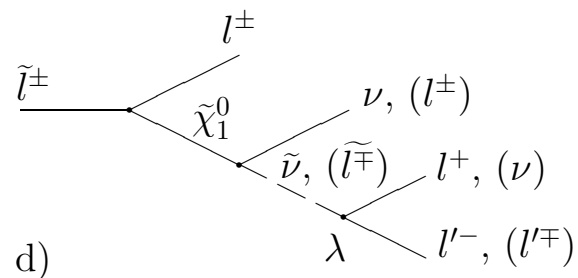

Figure 3: Sneutrino and charged slepton decay diagrams. a: $\tilde{\nu}$ direct decay; b: $\tilde{l}$ direct decay; in these diagrams the $\lambda$ indicates the $R_{p}$ vertex. c: $\tilde{\nu}$ indirect decay via a $\tilde{\chi}^{0}$; d: $\tilde{l}$ indirect decay via a $\widetilde{\chi}^{0}$. 

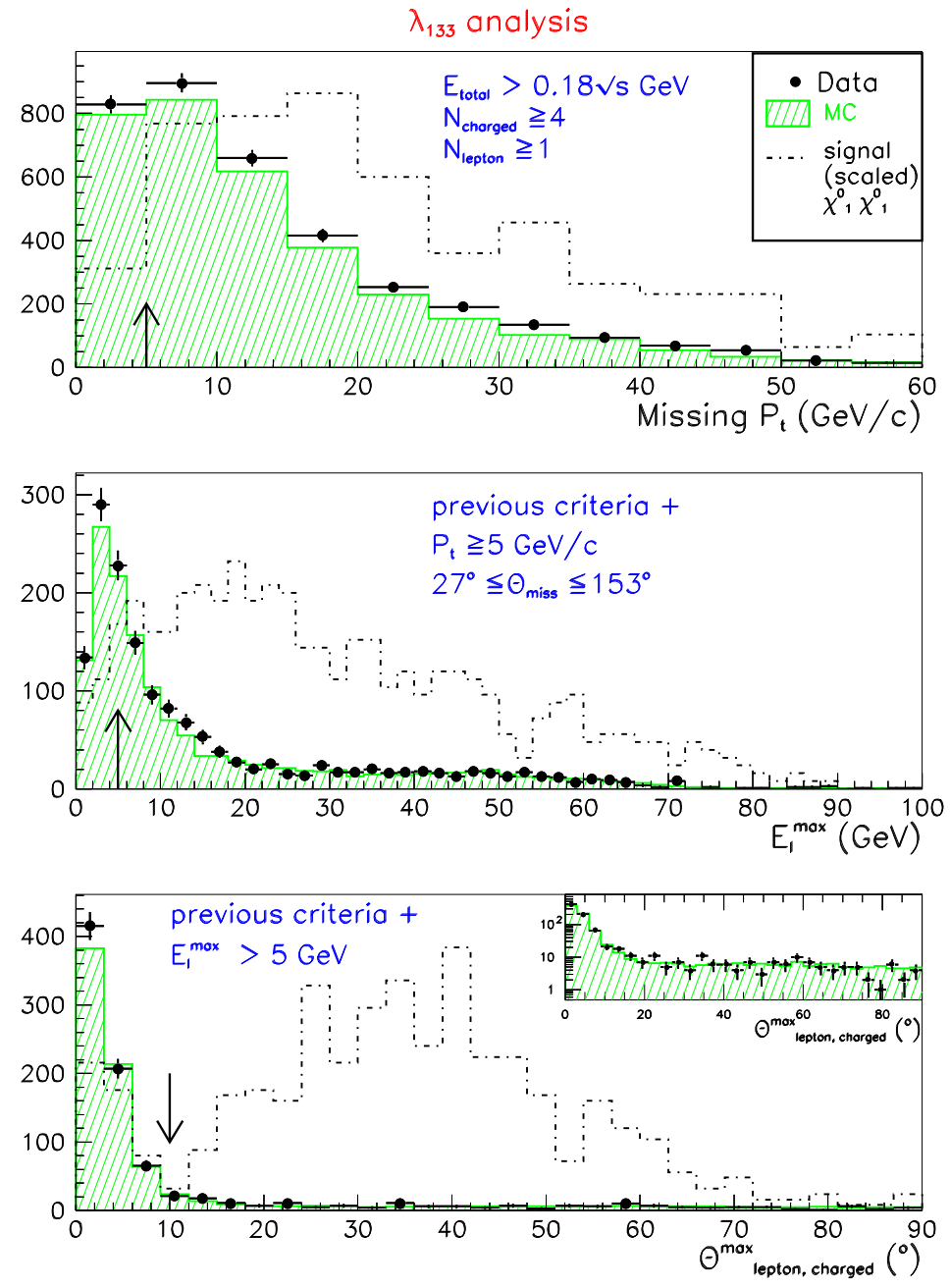

Figure 4: Neutralino and chargino search with the $\lambda_{133}$ coupling dominant: missing transverse momentum, energy of the most energetic lepton and isolation angle distributions for the data (black dots), expected background from standard model processes (hatched) and $\widetilde{\chi}_{1}^{0} \widetilde{\chi}_{1}^{0}$ signal (dotted line) generated for $\mathrm{m}_{\widetilde{\chi}_{0}^{1}}=34 \mathrm{GeV} / c^{2}$. The signal distribution has been scaled (see text) in order to be visible. The arrows show the applied cuts.

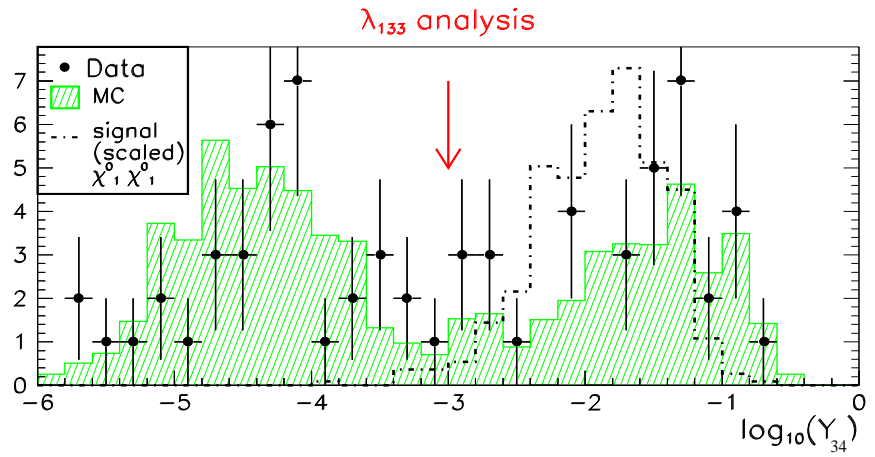

Figure 5: Neutralino and chargino search with a dominant $\lambda$ coupling: $\log _{10}\left(Y_{34}\right)$ distribution for the data (black dots) and the expected background from standard model processes (hatched histogram). A scaled signal distribution (generated for $\mathrm{m}_{\widetilde{\chi}_{0}^{1}}=34 \mathrm{GeV} / c^{2}$ ) is also plotted to show that the applied cut removes less than $1 \%$ of the signal, and half of the background. 

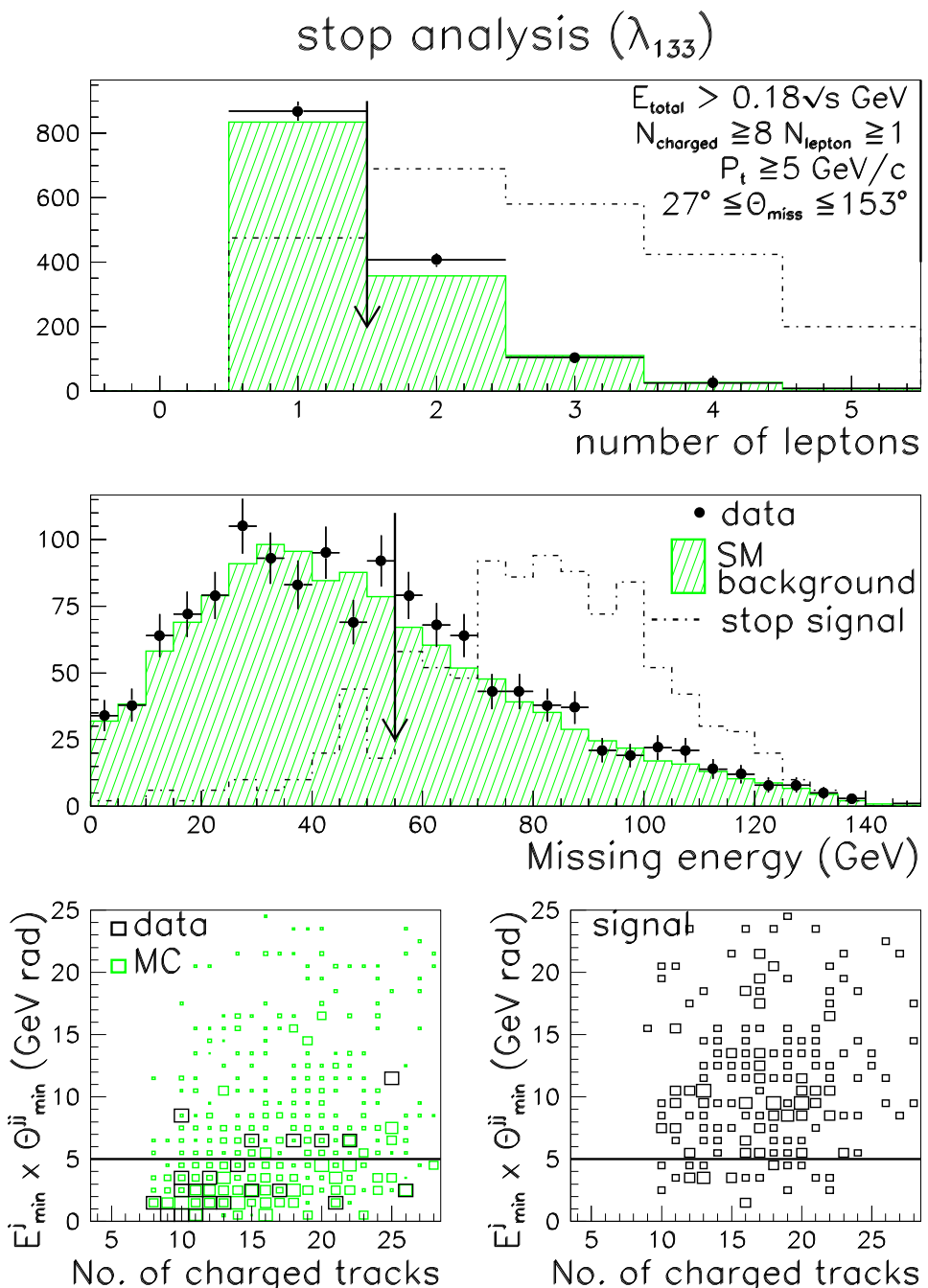

Figure 6: Stop indirect decay with the $\lambda_{133}$ coupling dominant. The two upper plots show the number of leptons and the missing energy distributions for real data (black dots), expected background from standard model processes (hatched) and $\tilde{t} \tilde{t}$ signal (dotted line) obtained during the preselection procedure; the arrows indicate the cut values. The signal has been scaled by an arbitrary factor in order to be visible. The two lower plots show the value of $E_{\min }^{j} \times \theta_{\min }^{j_{1}, j_{2}}$ versus the number of charged particles for the data and the background from standard model processes (on the left) and for the signal (on the right) after the cut $N_{\text {lepton }} \geq 2$; the horizontal line illustrates the cut. The signal has been generated for $\mathrm{m}_{\tilde{t}_{R}}=65 \mathrm{GeV} / c^{2}$ 
Neutralino and chargino searches at $\sqrt{ } s=183 \mathrm{GeV}$
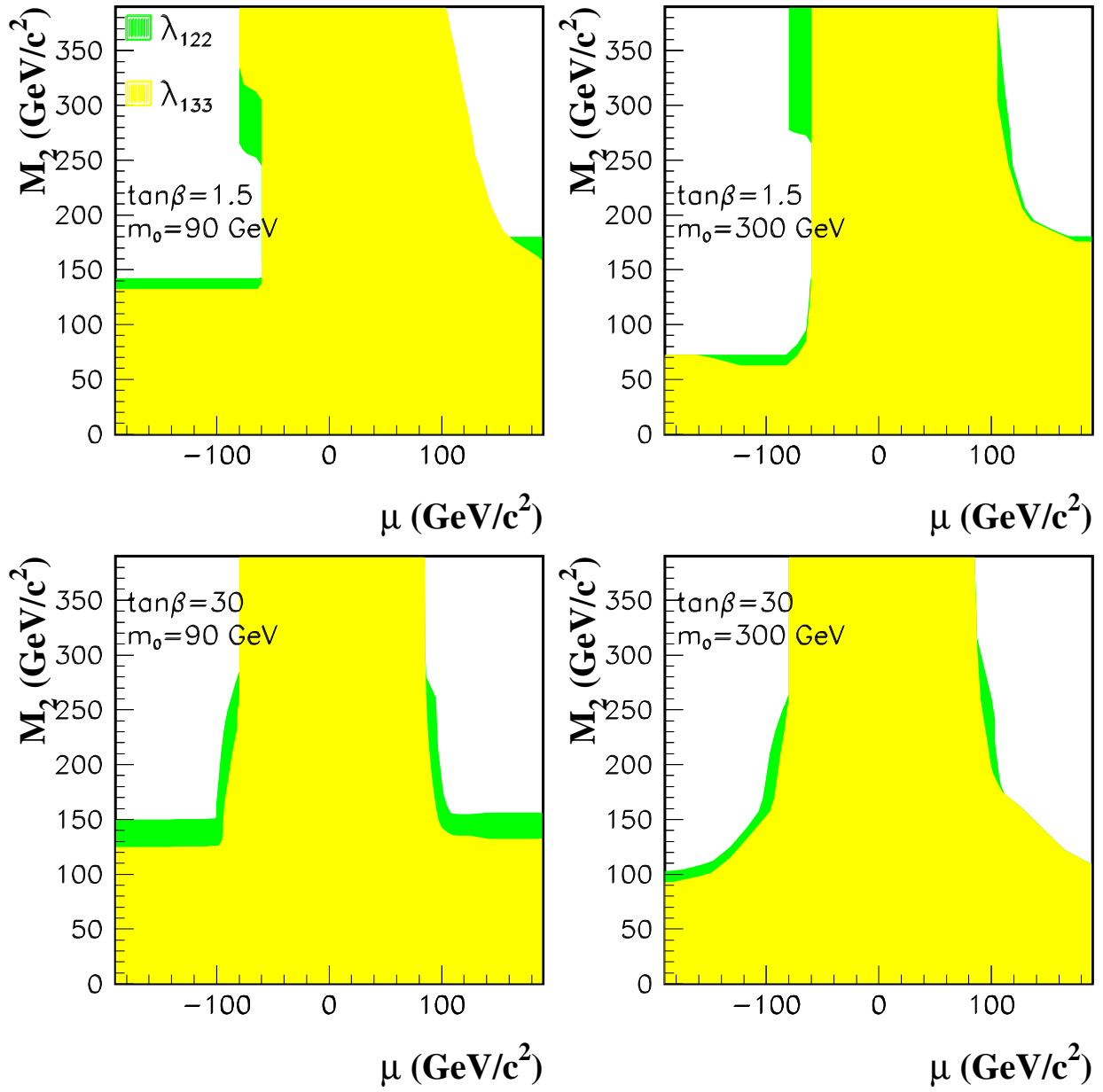

Figure 7: Neutralino and chargino searches in DELPHI data at $183 \mathrm{GeV}$ with a dominant $\lambda$ coupling: regions in $\mu, M_{2}$ parameter space excluded at $95 \%$ C.L. for two values of $\tan \beta$ and two values of $m_{0}$. The exclusion area obtained from the $\lambda_{133}$ search is shown in light grey and the corresponding area for the $\lambda_{122}$ search is shown in dark grey. The second exclusion area includes the first. 


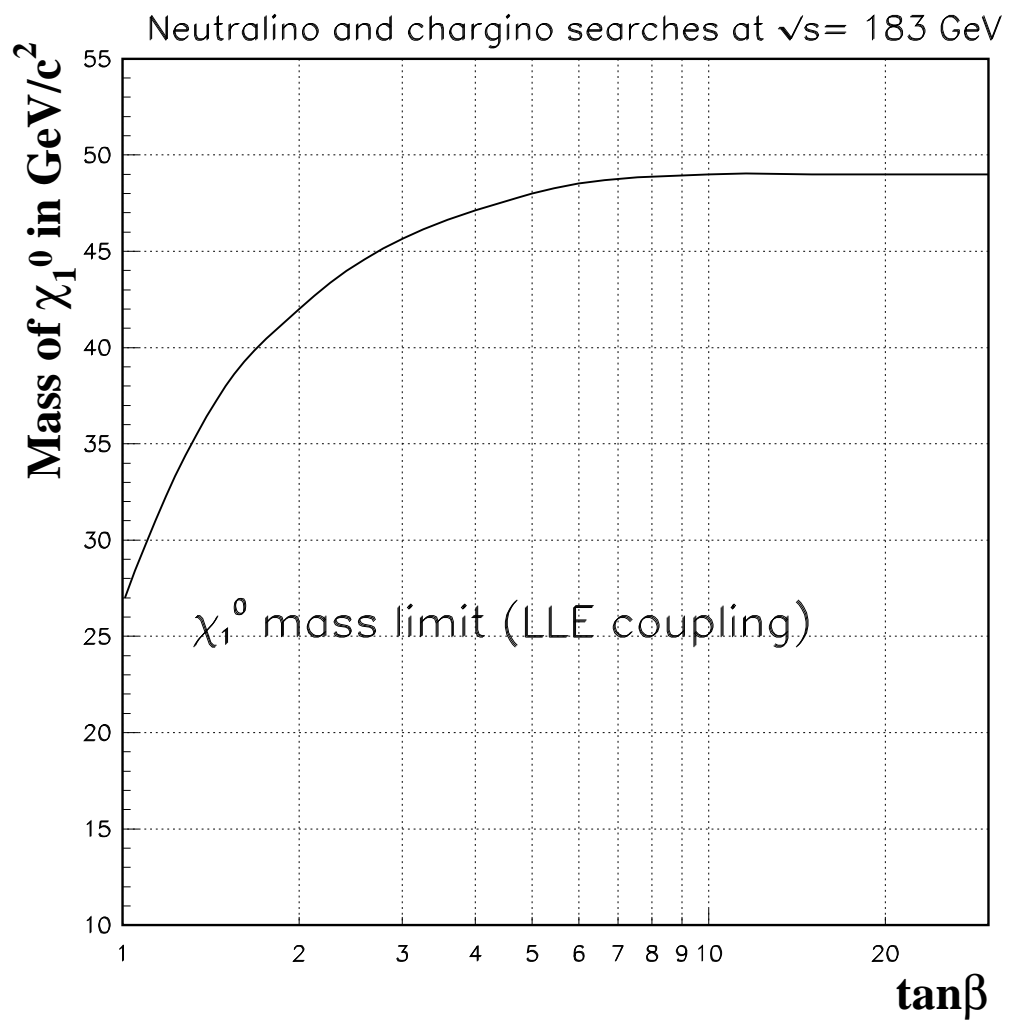

Figure 8: The lightest neutralino mass as a function of $\tan \beta$ at $95 \%$ confidence level. This limit is independent of the choice of $m_{0}$ in the explored range and of the generation indices $i, j, k$ of the $\lambda_{i j k}$ coupling. 
Sneutrino direct decay searches at $\sqrt{ } s=183 \mathrm{GeV}$

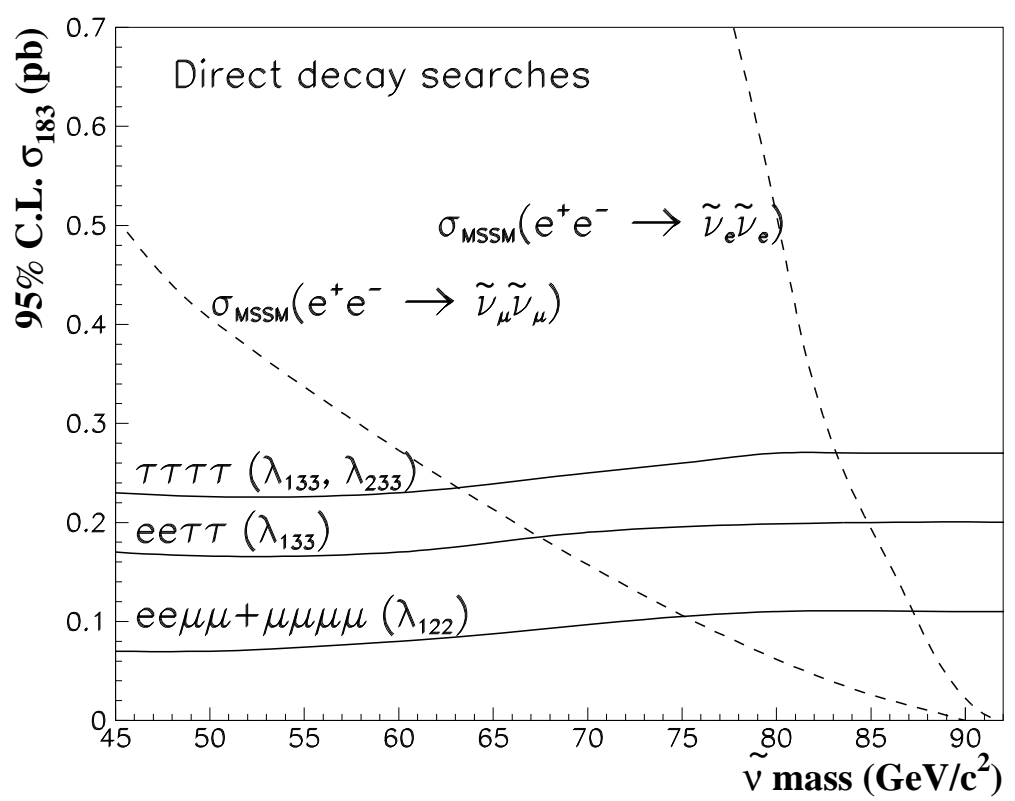

Figure 9: Sneutrino direct decay with $\lambda$ coupling: the limit on the $\tilde{\nu} \tilde{\bar{\nu}}$ production cross-section as a function of the mass or different final states. The MSSM cross-sections are reported, in order to derive a limit on the sneutrino mass in the case of direct $R_{p}$ decay. The dashed upper curve on the plot is the $\widetilde{\nu}_{\mathrm{e}} \widetilde{\bar{\nu}}_{\mathrm{e}}$ cross-section obtained for $\mu=-200 \mathrm{GeV} / c^{2}$ and $M_{2}=100 \mathrm{GeV} / c^{2}$, the corresponding chargino mass lies between 90 and $120 \mathrm{GeV} / c^{2}$.

Sneutrino searches at $\sqrt{ } \mathrm{s}=183 \mathrm{GeV}$

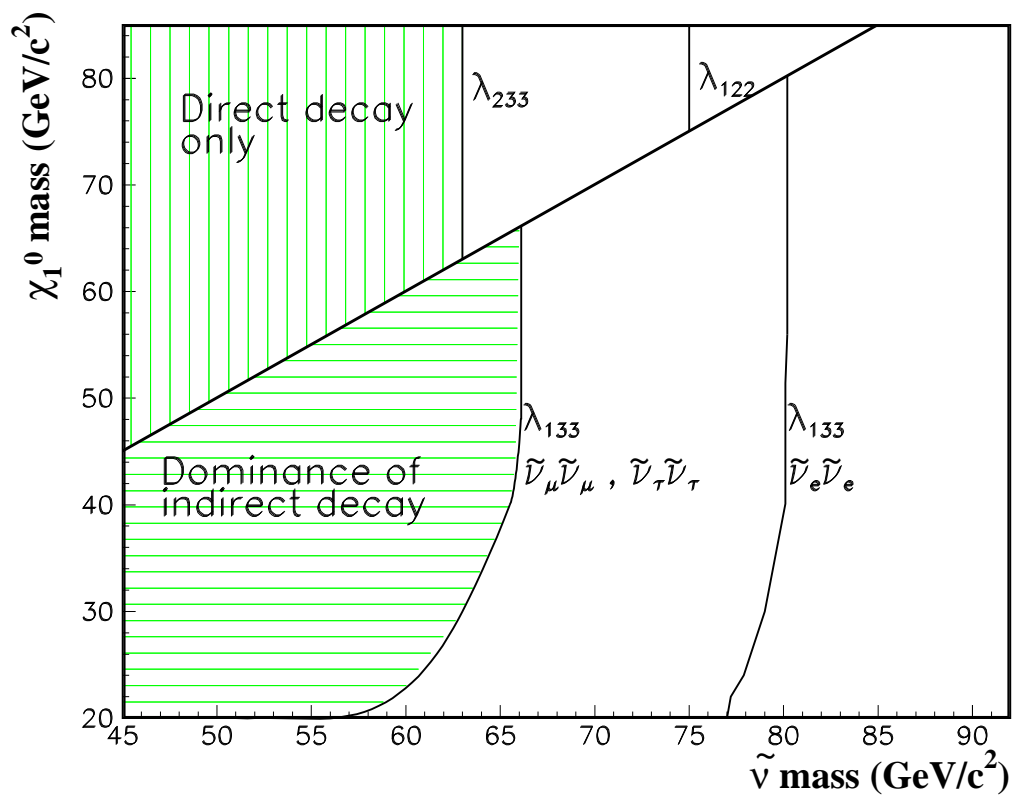

Figure 10: Sneutrino search with $\lambda$ coupling: exclusion domain in $\mathrm{m}_{\tilde{\chi}^{0}}$ versus $\mathrm{m}_{\tilde{\nu}}$ for $\tilde{\nu}$ pair production cross-section; the diagonal line separates the plot into two regions: in the upper part, only the direct decay is allowed; in the lower part, the indirect decay is dominant, so the exclusion limit also depends on the neutralino mass. In both cases, only the most conservative limit is shown for $\widetilde{\nu}_{\mu}$ and $\widetilde{\nu}_{\tau}$ production, and for the $\widetilde{\nu}_{\mathrm{e}}$ in case of chargino mass close to the kinematic limit. 
Charged slepton direct decay searches at $\sqrt{ } s=183 \mathrm{GeV}$

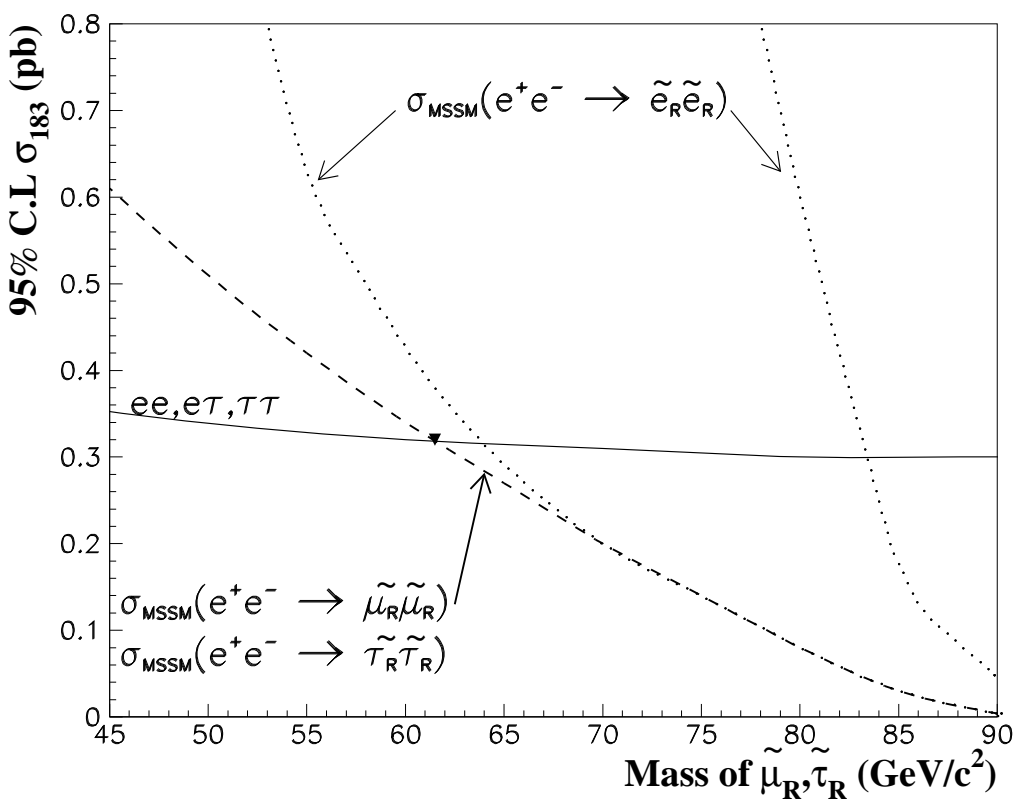

Figure 11: Slepton direct decay with $\lambda$ coupling: the full line shows the limit on the $\widetilde{l l}$ cross-section as a function of the slepton mass. The dashed curve gives the MSSM cross-section for $\tilde{\mu} \tilde{\mu}, \tilde{\tau} \tilde{\tau}$ production. The two dotted curves show the bounds of the ẽe cross-section since it depends on the contribution of the neutralino exchange in the $t$-channel (lower dotted curve obtained for $\tan \beta=1.01, \mu=-200 \mathrm{GeV} / c^{2}$ and $m_{0}=20 \mathrm{GeV} / c^{2}$, upper dotted curve obtained for $\tan \beta=30, \mu=-200 \mathrm{GeV} / c^{2}$ and $\left.m_{0}=60 \mathrm{GeV} / c^{2}\right)$.

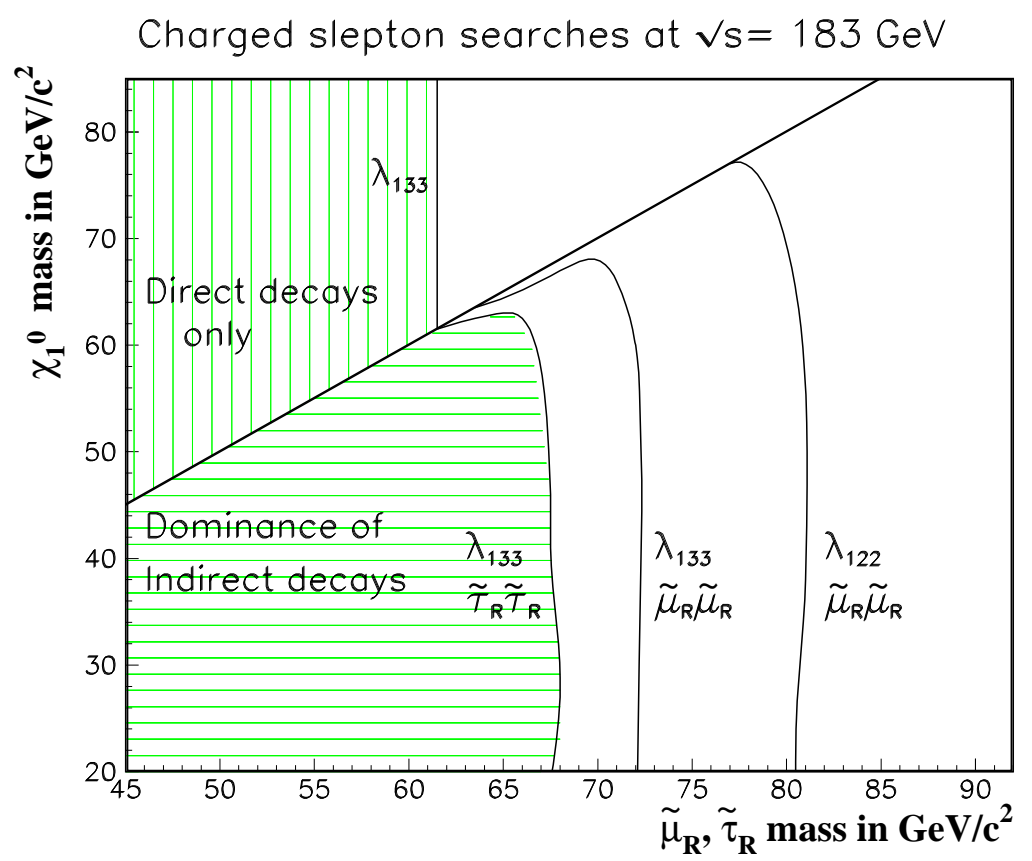

Figure 12: Slepton search with $\lambda$ coupling: exclusion domain in $m_{\tilde{\chi}^{0}}$ versus $m_{\tilde{l}}$ for the $\widetilde{l}$ pair production cross-section; the diagonal line separates the plot into two regions: in the upper part, only the direct decay is allowed; in the lower part, the indirect decay is dominant, so the exclusion limit depends also on the neutralino mass. The limit is given by the direct decay. 


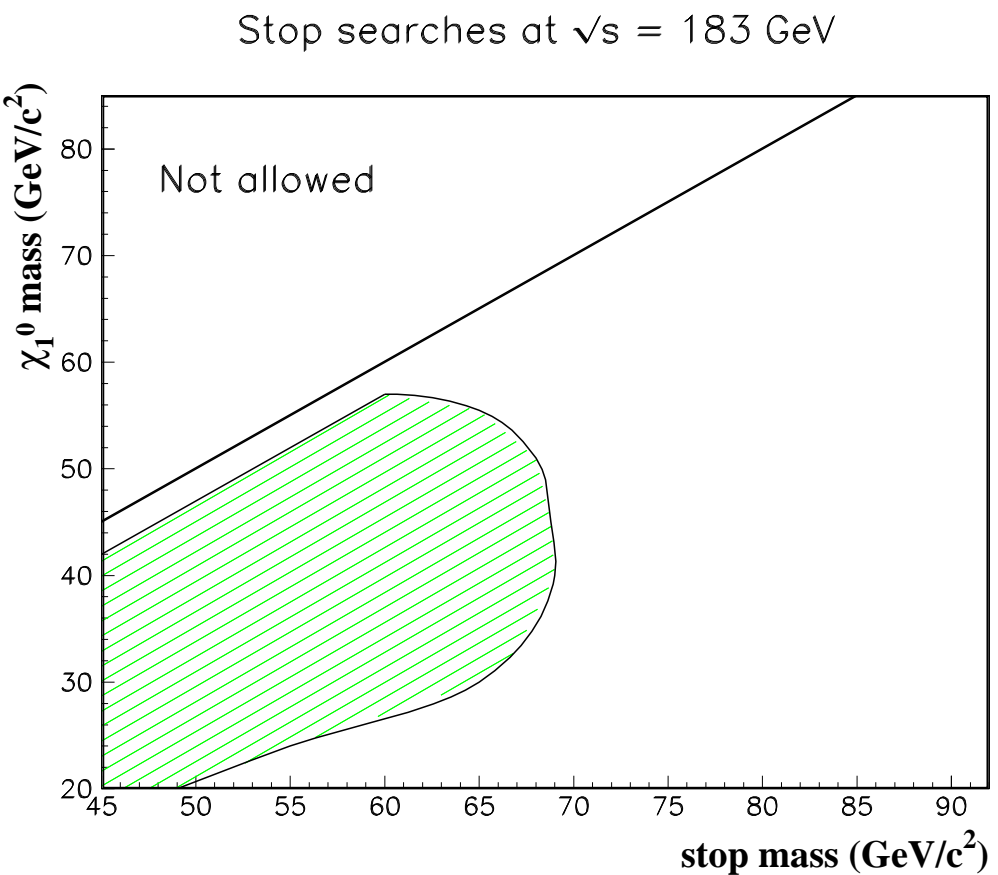

Figure 13: Stop indirect decay with $\lambda$ coupling: exclusion domain in $m_{\tilde{\chi}^{0}}$ versus $m_{\tilde{t}}$ for the $\tilde{t}_{1}$ pair production in case of maximal decoupling to the $\mathrm{Z}$ boson; the diagonal line separates the plot into two regions: in the upper part, no $R_{p}$ decay of $\tilde{t}$ is allowed; in the lower part, the indirect decay into c $\widetilde{\chi}_{1}^{0}$ is allowed, so the excluded area (hatched part) depends on the neutralino mass. 